\title{
Nonlinear Numerics
}

by EUSEBIUS J. DOEDEL

Concordia University, Montreal, Canada

ABSTRACT: The objectives and some basic methods of numerical bifurcation analysis are described. Several computational examples are used to illustrate the power as well as the limitations of these techniques. Future directions of algorithmic and software development are also discussed.

\section{Introduction}

The principal objective of numerical bifurcation analysis, as opposed to numerical simulation, is to compute continuum families of solutions to well-defined operator equations. Such computational results give a deeper understanding of the solution behavior, stability, multiplicity, and bifurcations, and they often provide direct links to the underlying mathematical theories.

The basic numerical method is continuation, the language in which algorithms are typically expressed and analyzed is functional analysis, and the fundamental theoretical tool is often the Implicit Function Theorem (IFT). For ordinary differential equations (ODEs), the numerical algorithms have attained a high degree of reliability. For introductions see Rheinboldt (1), Seydel (2), and Doedel et al. $(3,4)$, and for a comprehensive literature overview see Allgower and Georg (5).

Here we highlight some representative basic algorithms used in the numerical analysis of nonlinear equations. The emphasis is on ODEs, for which there exist several software packages in which these techniques have been implemented. We describe computational results for four different problems, namely, a singular perturbation problem, a problem with "bursting" phenomena (Plant's model), a problem with derivative discontinuities (Chua's circuit), and a problem with homoclinic bifurcations (coupled Josephson junctions). These examples are used to illustrate the power as well as the limitations of the numerical techniques.

We conclude by describing a selection of problems which we think merit future work, since resulting algorithms and software would find many applications. Some current efforts in these directions are indicated.

\section{Continuation}

\subsection{Regular solution points}

Consider the equation $G(x)=0$, where $G: R^{n+1} \rightarrow R^{n}$. Generically, there will be curves ("branches") of solutions, which can be effectively computed by pseudo-arclength 
continuation (6). Given a solution $x_{0}$, say, and the direction of the solution branch, $\dot{x}_{0}$, a nearby solution $x_{1}$ is computed by solving

$$
\begin{gathered}
G\left(x_{1}\right)=0, \\
\left(x_{1}-x_{0}\right)^{*} \dot{x}_{0}=\Delta s,
\end{gathered}
$$

for $x_{1}$. Typically Newton's method is used, and the next direction vector $\dot{x}_{1}$ is then easily computed as a by-product. It is easy to see why and when this continuation technique works.

In a more general setting, suppose that $G\left(x_{0}\right)=0$, where $G: \mathscr{B}_{1} \rightarrow \mathscr{B}_{2}$. Here $\mathscr{B}_{1}$ and $\mathscr{B}_{2}$ are appropriate Banach spaces. Suppose that $x_{0}$ is a regular solution point, i.e.

$$
\mathscr{N}\left(G_{x}^{0}\right)=\operatorname{Span}\{\varphi\}, \mathscr{N}\left(G_{x}^{0 *}\right)=\{0\},
$$

where $G_{x}^{0 *}$ is the adjoint of $G_{x}^{0}$. Consider again the extended system

$$
\mathscr{F}(x) \equiv\left\{\begin{array}{c}
G(x) \\
\left(x-x_{0}\right) * \varphi-s .
\end{array}\right\}
$$

\section{Theorem}

Near a regular solution point $x_{0}$, the equation $\mathscr{F}(x)=0$ defines a unique solution curve $x=x(s)$.

Proof: This follows from the IFT, since

$$
\mathscr{F}_{\mathrm{x}}^{0}=\left(\begin{array}{c}
G_{x}^{0} \\
\varphi^{*}
\end{array}\right)
$$

is nonsingular.

We note that, in some cases, numerical continuation computations can be rigorous; see, for example, Kearfott (7).

\subsection{Simple singular points}

Next suppose that $G\left(x_{0}\right)=0$, where, again, $G: \mathscr{B}_{1} \rightarrow \mathscr{B}_{2}$. Suppose that $x_{0}$ is a simple singular point, i.e.

$$
\mathscr{N}\left(G_{x}^{0}\right)=\operatorname{Span}\left\{\varphi_{1}, \varphi_{2}\right\}, \mathscr{N}\left(G_{x}^{0 *}\right)=\operatorname{Span}\{\psi\} .
$$

Consider the equations

$$
F\left(y, \mu ; \epsilon_{1}, \epsilon_{2}\right) \equiv\left\{\begin{array}{c}
G\left(x_{0}+\epsilon_{1} \varphi_{1}+\epsilon_{2} \varphi_{2}+y\right)+\mu \psi=0, \\
y^{*} \varphi_{1}=0, \\
y^{*} \varphi_{2}=0 .
\end{array}\right\}
$$

We have $F(0,0 ; 0,0)=0$.

\section{Theorem}

Near $\left(y, \mu ; \epsilon_{1}, \epsilon_{2}\right)=(0,0 ; 0,0)$, the equation $F\left(y, \mu ; \epsilon_{1}, \epsilon_{2}\right)=0$ defines a unique solution manifold 


$$
y=y\left(\epsilon_{1}, \epsilon_{2}\right), \mu=\mu\left(\epsilon_{1}, \epsilon_{2}\right),
$$

with $y(0,0)=0, \mu(0,0)=0$.

Proof: This also follows from the IFT, since the Fréchet derivative

$$
\left(F_{y}\left(y, \mu ; \epsilon_{1}, \epsilon_{2}\right) \mid F_{\mu}\left(y, \mu ; \epsilon_{1}, \epsilon_{2}\right)\right.
$$

at $\left(y, \mu ; \epsilon_{1}, \epsilon_{2}\right)=(0,0 ; 0,0)$, given by

$$
\left(\begin{array}{ll}
G_{x}^{0} & \psi \\
\varphi_{1}^{*} & 0 \\
\varphi_{2}^{*} & 0
\end{array}\right)
$$

is nonsingular.

Assuming $F$ (i.e. $G$ ) to be sufficiently smooth, we can derive the Taylor expansion

$$
\mu\left(\epsilon_{1}, \epsilon_{2}\right)=\frac{1}{2} \frac{\psi^{*} G_{x x}^{0} \varphi_{1} \varphi_{1}}{\psi^{*} \psi} \epsilon_{1}^{2}+\frac{\psi^{*} G_{x x}^{0} \varphi_{1} \varphi_{2}}{\psi^{*} \psi} \epsilon_{1} \epsilon_{2}+\frac{1}{2} \frac{\psi^{*} G_{x x}^{0} \varphi_{2} \varphi_{2}}{\psi^{*} \psi} \epsilon_{2}^{2}+O\left(\epsilon^{3}\right),
$$

and similarly for $y\left(\epsilon_{1}, \epsilon_{2}\right)$. To lowest order approximation, the solutions of $G(x)=0$ then correspond to the level curves

$$
\left(\psi^{*} G_{x x}^{0} \varphi_{1} \varphi_{1}\right) \epsilon_{1}^{2}+2\left(\psi^{*} G_{x x}^{0} \varphi_{1} \varphi_{2}\right) \epsilon_{1} \epsilon_{2}+\left(\psi^{*} G_{x x}^{0} \varphi_{2} \varphi_{2}\right) \epsilon_{2}^{2}=0 .
$$

This is the Algebraic Bifurcation Equation (ABE; see Keller (6)) from which, in the case of distinct real roots, two distinct directions

$$
x \approx x_{0}+\epsilon_{1} \varphi_{1}+\epsilon_{2} \varphi_{2}
$$

of bifurcating branches can be computed.

The above procedure, basically the Lyapunov-Schmidt procedure (8), also applies to higher-order singular points, and is the first step in the reduction to normal form. Normal forms are important in understanding the types of solutions that can exist near a singular point. However, these solutions often exist only in a very small neighborhood; see, for example, Wittenberg and Holmes (9). Normal forms are therefore of little use in determining the actual global behavior of a given system. For numerical purposes the importance of the above theorem lies in the ABE that it produces. Furthermore, the basic idea, namely that of "extended system", is at the heart of most techniques for regularizing singular computational problems. In fact, the pseudo-arclength continuation algorithm is the simplest instance of this idea.

\subsection{Linear algebra}

Extended systems for computing and continuing singular points often give matrices in Newton's method that have a special structure which can be exploited. A general presentation of the numerical linear algebra aspects of extended systems can be found in Govaerts (10). An example of a system with such a special structure is

$$
\left(\begin{array}{ccc}
A & O & c \\
C & A^{*} & 0 \\
0^{*} & \psi^{*} & 0
\end{array}\right)\left(\begin{array}{l}
x \\
y \\
z
\end{array}\right)=\left(\begin{array}{l}
f \\
g \\
h
\end{array}\right),
$$


where the $n+1$ by $n+m$ matrix $A$ has rank $n$, and where $A^{*}$ is the transpose of $A$. This system arises in the continuation of folds $(m=1)$, simple singular points $(m=2)$, and more generally in the continuation of $m$-parameter optima of some objective functional on a solution manifold $(3, \mathbf{4})$.

We now illustrate a solution procedure for the above system that requires only $L U$ decomposition (11) of the matrix $A$. Pivoting should be used. We have

$$
\mathscr{N}(A)=\operatorname{Span}\left\{\varphi_{i}\right\}_{i=1}^{m}, \mathscr{N}\left(A^{*}\right)=\operatorname{Span}\{\psi\} .
$$

The first equation is $A x=f-z c$. To be solvable, we must have

$$
z=\frac{\psi^{*} f}{\psi^{*} c}
$$

The solution component $x$ then has the form $x=x_{p}+\sum_{i=1}^{m} \alpha_{i} \varphi_{i}$. Here $x_{p},\left\{\varphi_{i}\right\}_{i=1}^{m}$, and $\psi$ can easily be computed once $A$ has been $L U$-decomposed.

From the second equation it follows that $\varphi_{i}^{*} C\left(x_{p}+\sum_{j=1}^{m} \alpha_{j} \varphi_{j}\right)=\varphi_{i}^{*} g$, for $i=1, \ldots, m$, or

$$
\sum_{j=1}^{m}\left(\varphi_{i}^{*} C \varphi_{j}\right) \alpha_{j}=\varphi_{i}^{*} g-\varphi_{i}^{*} C x_{p}, \quad i=1, \ldots, m
$$

which we solve for the $\left\{\alpha_{i}\right\}_{i=1}^{m}$. Then

$$
x=x_{p}+\sum_{i=1}^{m} \alpha_{i} \varphi_{i}
$$

Now find a particular solution $y_{p}$ to $A^{*} y=g-C x$, for which the $L U$-decomposition of $A$ can be used. Finally we have $y=y_{p}+\beta \psi$, where, by the third equation, $\psi^{*}\left(y_{p}+\beta \psi\right)=h$, from which

$$
\beta=\frac{h-\psi^{*} y_{p}}{\psi^{*} \psi}
$$

This solution algorithm also establishes the nonsingularity of the full matrix, given that $A$ has rank $n$, that $c \notin \mathscr{R}(A)$, and provided that the matrix $Q$, with entries $Q_{i, j} \equiv \varphi_{i}^{*} C \varphi_{j}$, is nonsingular. These conditions are generically satisfied.

\section{Numerical bifurcation analysis of ODEs}

Here we describe some basic ODE continuation problems and we discuss a particularly suitable discretization technique, namely orthogonal collocation.

\subsection{Boundary value problems}

The ODE boundary value problem addressed in Doedel et al. $(3,4)$ is of the form

$$
u^{\prime}(t)=f(u(t), \lambda), \quad t \in[0,1], u(\cdot)_{2} f(\cdot, \cdot) \in R^{n}, \lambda \in R^{n_{i}},
$$

with boundary conditions 


$$
b_{i}(u(0), u(1), \lambda)=0, i=1,2, \ldots, n_{b},
$$

and integral constraints

$$
\int_{0}^{1} q_{i}(u(t), \lambda) \mathrm{d} t=0, i=1,2, \ldots, n_{q},
$$

where we assume that $n_{i}=n_{b}+n_{q}-n+1$, which generically leads to one-dimensional continua ("branches") of solutions.

\subsection{Periodic solutions}

A practically important example of a system of the above form arises from the numerical continuation of periodic solutions. A numerical scheme for computing stable and unstable periodic solutions is given by the time-scaled ODE

$$
u^{\prime}(t)=T f(u(t), \lambda),
$$

where $T$ denotes the actual period, subject to the periodicity condition $u(0)=u(1)$, and a phase condition

$$
\int_{0}^{1} u(t)^{*} \hat{u}^{\prime}(t) \mathrm{d} t=0
$$

This integral constraint, where $\hat{u}$ is a nearby reference solution, is a necessary condition for $D(\sigma) \equiv \int_{0}^{1}\|u(t+\sigma)-\hat{u}(t)\|^{2} \mathrm{~d} t$ to be minimized over $\sigma$. After appropriate discretization, solutions of the above equations can be continued using the pseudo-arclength continuation technique. Asymptotic stability and bifurcation information can be extracted from the linearized Newton systems.

\subsection{Connecting orbits}

Another ODE boundary value system arises from the numerical continuation of connecting orbits, i.e. orbits that connect fixed points of a vector field. The basic algorithm, which can be presented in various forms $(12,13,14)$, consists of continuation of solutions to the following set of equations: the time-scaled differential equations

$$
u^{\prime}(t)=T f(u(t), \lambda), u(\cdot), f(\cdot, \cdot) \in R^{n}, \lambda \in R^{n_{\lambda}},
$$

the stationary point equations

$$
f\left(w_{0}, \lambda\right)=0, f\left(w_{1}, \lambda\right)=0,
$$

and the eigenvalue-eigenvector and normalization relations

$$
\begin{aligned}
& f_{u}\left(w_{0}, \lambda\right) v_{0 i}=\mu_{0 i} v_{0 i}, v_{0 i}^{*} v_{0 i}=1, i=1, \ldots, n_{0}, \\
& f_{u}\left(w_{1}, \lambda\right) v_{1 i}=\mu_{1 i} v_{1 i}, v_{1 i}^{*} v_{1 i}=1, i=1, \ldots, n_{1},
\end{aligned}
$$

where, for simplicity, we assume that $f_{u}\left(w_{0}, \lambda\right)$ has $n_{0}$ distinct real positive eigenvalues $\mu_{0 i}$ with eigenvectors $v_{0 i}$, and that $f_{u}\left(w_{1}, \lambda\right)$ has $n_{1}$ distinct real negative eigenvalues $\mu_{1 i}$ with eigenvectors $v_{1 i}$. (In practice, it is better to work with an orthogonal basis of the corresponding invariant subspaces $E_{0}$ and $E_{1}$, or their orthogonal complements.) 
One can also add a phase condition

$$
\int_{0}^{1}(f(u, \lambda)-f(\hat{u}, \hat{\lambda}))^{*} f_{u}(\hat{u}, \hat{\lambda}) f(\hat{u}, \hat{\lambda}) \mathrm{d} t=0 .
$$

This integral constraint, with reference solution $(\hat{u}, \hat{\lambda})$, is a necessary condition for minimizing $D(\sigma) \equiv \int_{0}^{1}\left\|u^{\prime}(t+\sigma)-\hat{u}^{\prime}(t)\right\|^{2} \mathrm{~d} t$ over $\sigma$, also using the fact that $u^{\prime \prime}(t)=f_{u}(u, \lambda) u^{\prime}(t)=f_{u}(u, \lambda) f(u, \lambda)$. Finally, we require $u(0)$ to lie in the tangent manifold $E_{0}$ at "distance" $\epsilon_{0}$ from $w_{0}$ and, similarly, $u(1)$ in $E_{1}$ at distance $\epsilon_{1}$ from $w_{1}$ :

$$
\begin{aligned}
& u(0)=w_{0}+\epsilon_{0} \sum_{i=1}^{n_{0}} c_{0 i} v_{0 i}, \sum_{i=1}^{n_{0}} c_{0 i}^{2}=1, \\
& u(1)=w_{1}+\epsilon_{1} \sum_{i=1}^{n_{1}} c_{1 i} v_{1 i}, \sum_{i=1}^{n_{1}} c_{1 i}^{2}=1 .
\end{aligned}
$$

These last equations can be used to eliminate $w_{0}$ and $w_{1}$ from the earlier equations. This leaves $n$ coupled differential equations subject to $n_{c} \equiv 2 n+(n+1)\left(n_{0}+n_{1}\right)+3$ constraints. In addition to $u(t) \in R^{n}$ we have scalar variables $\lambda \in R^{n_{i}}, \epsilon_{0}, \epsilon_{1} \in R, \mu_{0 i}, c_{0 i} \in R$, $v_{0} \in R^{n}$, for $i=1, \ldots, n_{0}$, and $\mu_{1 i}, c_{1} \in R, v_{1} \in R^{n}$, for $i=1, \ldots, n_{1}$. The total number of scalar variables is $n_{v} \equiv n_{\lambda}+(n+2)\left(n_{0}+n_{1}\right)+2$. Generically we need $n_{v}=n_{c}-n$. Thus the number of parameters required for a single heteroclinic connection, i.e. the codimension of the orbit, is $n_{\lambda} \equiv n-\left(n_{0}+n_{1}\right)+1$. A branch of orbits requires $n_{\lambda}+1$ free parameters. The integration time $T$ is "large" and fixed. It can be shown, under appropriate transversality conditions, that the above scheme is well-posed.

A particular case is the heteroclinic connection of two saddle points in $R^{2}$. Here $n=2$ and $n_{0}=n_{1}=1$, so $n_{\lambda}=1$. A branch of such orbits requires two problem parameters. Another example is the connection of a saddle to a node in $R^{2}$. Here $n=2, n_{0}=1$ and $n_{1}=2$, so $n_{i}=0$. A branch of such connections requires one problem parameter. We also have the homoclinic orbit, for which $w_{0}=w_{1}$ and $n_{0}+n_{1}=n$, so that $n_{\lambda}=1$. Often such orbits can also be computed as the limit of periodic orbits as the period $T \rightarrow \infty$.

\subsection{Discretization}

There are now several software packages for the numerical bifurcation analysis of ODEs (see Allgower and Georg (5) for a survey), some of which are more convenient to use than others. However, it is generally recognized that those based on global solution techniques (as opposed to "shooting") and orthogonal collocation with piecewise polynomial functions (as opposed to standard finite differences) perform best on difficult problems. Here "difficult" refers to boundary or interior layers, strong relaxation phenomena, sharp fronts and similar phenomena. A selection of such problems will be presented in Section 4.

Introduce a mesh $\left\{0=t_{0}<t_{1}<\ldots<t_{N}=1\right\}$, and let $\Delta t_{j} \equiv t_{j}-t_{j}-1$ for $1 \leq j \leq N$. Define

$$
\mathscr{P}_{h}^{m}=\left\{p_{h} \in C[0,1]:\left.p_{h}\right|_{L_{j}, t_{j-1}} \in \mathscr{P}^{m}\right\},
$$

where $\mathscr{P}^{m}$ is the space of polynomials of degree less than or equal to $m$. The orthogonal 
collocation method with piecewise polynomials (15) consists of finding $p_{h} \in \mathscr{P}_{h}^{m}$ and $\lambda$ such that

$$
p_{h}^{\prime}\left(z_{j, i}\right)=f\left(p_{h}\left(z_{j, i}\right), \lambda\right), j=1, \ldots, N, i=1, \ldots, m,
$$

and such that $p_{h}$ satisfies the boundary and integral conditions, including the pseudoarclength equation. In each interval $\left[t_{j-1}, t_{j}\right]$ the collocation points $z_{j, i}$ are the transformed roots of the $m$ th orthogonal polynomial ("Gauss points").

This method gives a globally accurate solution, which is especially accurate at the main mesh points $t_{j}$ and for the scalar variables $\lambda$ ("superconvergence"; see de Boor and Swartz (16)). Moreover, there is a known "optimal" mesh selection strategy that minimizes the number of mesh intervals (15). This safeguards, to some extent, against spurious solutions and, combined with continuation, makes it easier to compute difficult solutions. The collocation method also makes it easy to handle integral constraints. Finally, the method leads to a sparse Jacobian matrix in Newton's method, which can be efficiently solved, allows the computation of Floquet multipliers at little extra expense (17), and gives reasonable performance when implemented on a parallel computing system (18).

\subsection{Collocation for PDEs}

Piecewise polynomial collocation has been extended to certain classes of partial differential equations (PDEs), but its use there has not been as widespread as for ODEs. Here we briefly describe a particular collocation method that may offer advantages in bifurcation software for systems of nonlinear elliptic PDEs. Consider

$$
\Delta u=f(u), x \in \Omega \subset R^{N},
$$

where $\Delta$ is the Laplace operator with appropriate boundary conditions and integral constraints. We suppress explicit indication of parameters. Suppose the domain is an $N$-cube, which has been recursively subdivided into $2^{M}$ subrectangles ("finite elements"). To each finite element associate appropriate boundary matching points $x_{i}$, for $i=1, \ldots, n$, interior collocation points $z_{i}$, for $i=1, \ldots, m$, and a polynomial $p(x)$ of "order" $n+m$. Require that, at the points $x_{i}$ on any common boundary, the values of neigboring polynomials and their normal derivatives match. Also require each polynomial to satisfy the collocation equations

$$
\Delta p\left(z_{i}\right)=f\left(p\left(z_{i}\right)\right), i=1, \ldots, m .
$$

\section{Example}

For the Laplace equation $\Delta u(x)=f(x)$ in $R^{2}$, with a uniform mesh, one central matching point per element boundary, and one central collocation point per element, an equivalent "finite difference" approximation will have the form

$$
v_{i}=\sum_{j=1}^{4} \alpha_{i j} u_{j}+\beta_{i} f(z)
$$

where $u_{i}$ and $v_{i}$ are the approximate solution and its normal derivative, respectively, at the matching point $x_{i}$. As a basis, choose $\left\{1, x, y, x^{2}, y^{2}\right\}$. Then, for each finite element, one obtains a discrete set of equations 


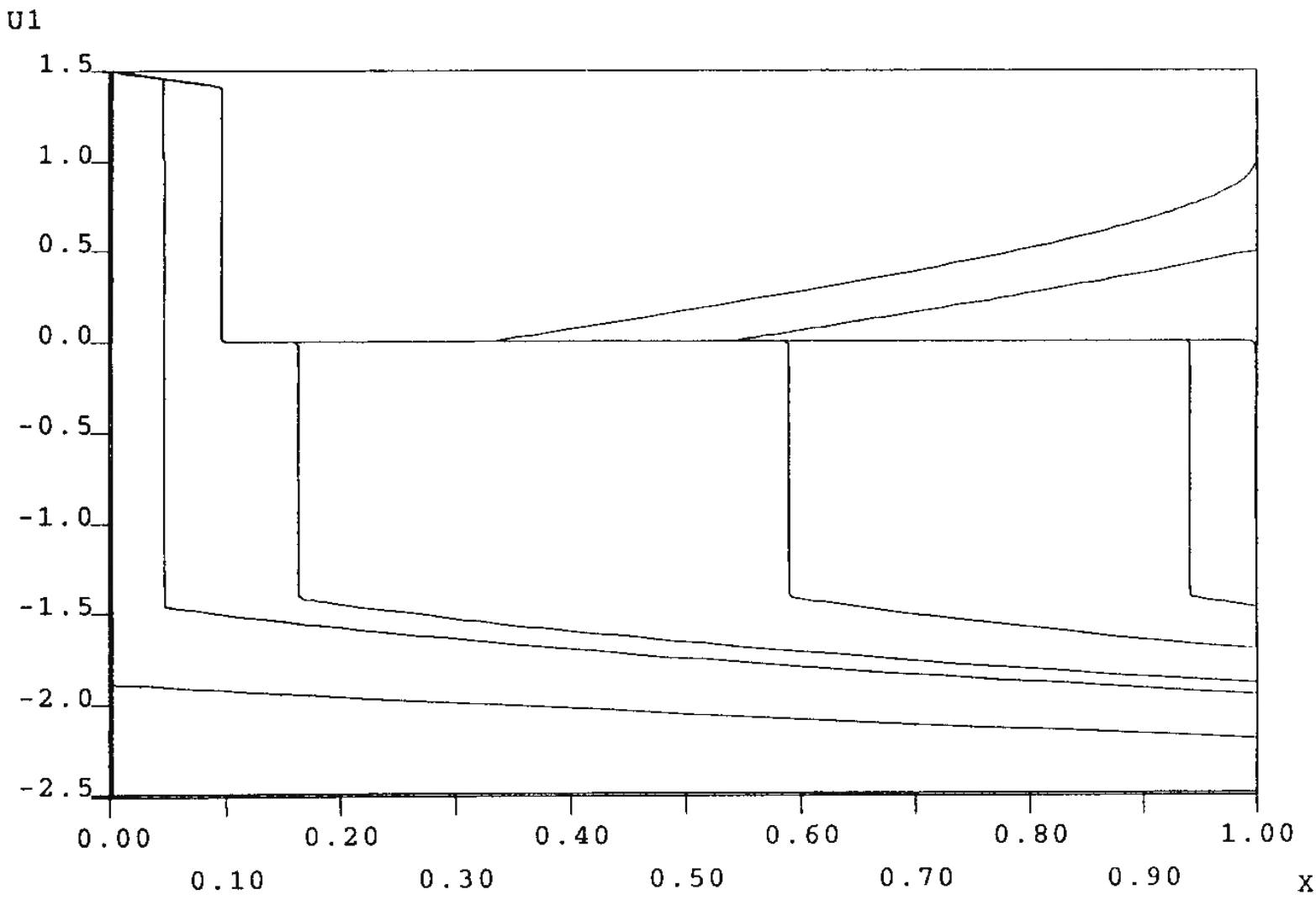

Fig. 1. Eight solutions to the singular perturbation problem. Six are qualitatively distinct.

$$
\begin{aligned}
& v_{1}=\left(2 u_{1}-u_{2}-u_{4}\right) / 2 h+h f(z) / 2, \\
& v_{2}=\left(2 u_{2}-u_{1}-u_{3}\right) / 2 h+h f(z) / 2, \\
& v_{3}=\left(2 u_{3}-u_{2}-u_{4}\right) / 2 h+h f(z) / 2, \\
& v_{4}=\left(2 u_{4}-u_{1}-u_{3}\right) / 2 h+h f(z) / 2 .
\end{aligned}
$$

This method is second-order accurate.

In its more general formulation, this PDE collocation method allows high-order accuracy and facilitates nested dissection, which reduces computational complexity (19).

\section{Computational examples}

\subsection{A singular perturbation problem}

Consider the ODE boundary value problem (20)

$$
\epsilon u^{\prime \prime}-u u^{\prime}\left(u^{2}-1\right)-u=0,
$$

with $u(0)=3 / 2, u(1)=\gamma$. Written as a system, this becomes

$$
\begin{aligned}
& u_{1}{ }^{\prime}=u_{2}, \\
& u_{2}{ }^{\prime}=\lambda\left[u_{1} u_{2}\left(u_{1}^{2}-1\right)+u_{1}\right] / \epsilon,
\end{aligned}
$$

with $u_{1}(0)=3 / 2, u_{1}(1)=\gamma$. The solution behavior for varying $\gamma$, which can be studied analytically, is illustrated in Fig. 1. There are six qualitatively different solutions for $\gamma$ in the range $[-5 / 2,3 / 2]$. The computations were done with $\epsilon=10^{-5}$, using orthogonal 
collocation with up to 200 mesh intervals, 4 Gauss collocation points per mesh interval, and (obviously) adaptive mesh selection. The artificial parameter $\lambda$ has been introduced for homotopy purposes. We start with $\lambda=0$, for which there is an exact solution, and with a moderate value of $\epsilon$, say, $\epsilon=1$. The first numerical continuation takes $\lambda$ from 0 to 1 . In the second continuation $\lambda=1$ remains fixed, while $\epsilon$ decreases from 1 to $10^{-5}$, say. The third continuation takes $\gamma$ from $3 / 2$ to $-5 / 2$, with both $\epsilon$ and $\lambda$ fixed. This final continuation is able to locate all different solution types that occur in the indicated range of $\gamma$ values. The calculations resolve the interior layers (in $x$ ), follow them as $\gamma$ changes, and determine the $\gamma$-ranges where the transitions occur.

Although these calculations show the effectiveness of the continuation and discretization algorithms, it must be noted that the calculation with $\epsilon=10^{-5}$ requires a very large amount of computer time. This is especially true in the range where an interior front moves with $\gamma$, and where the pseudo-arclength step must be very small in order for the Newton iterations to converge. For larger $\epsilon$, say $\epsilon=10^{-3}$, the calculations are relatively fast.

\subsection{Plant's model of bursting nerve cells}

A system is said to exhibit bursting when it changes back and forth between a quiescent state and a rapidly oscillating state. A five-variable ODE model for bursting in nerve cells developed by Plant (21) is given by

$$
\begin{aligned}
\dot{V} & =\left[g_{I} s_{I}^{3}(V) y_{I}+g_{T} x_{T}\right]\left[V_{I}-V\right]+\left[g_{K} x_{K}^{4}+g_{P} c\left(K_{p}+c\right)^{-1}\right]\left[V_{K}-V\right]+g_{L}\left[V_{L}-V\right], \\
\dot{x}_{T} & =\left[s_{T}(V)-x_{T}\right] /\left(\zeta \tau_{x T}\right), \\
\dot{x}_{K} & =\left[s_{K}(V)-x_{K}\right] /\left(\zeta \tau_{x K}\right), \\
\dot{y}_{I} & =\left[z_{I}(V)-y_{I}\right] /\left(\zeta \tau_{y I}\right), \\
\dot{c} & =\rho\left[K_{c} x_{T}\left(V_{C a}-V\right)-c\right] .
\end{aligned}
$$

Detailed expressions for the various functions in this model and for parameter values can be found in Plant's paper. We let $g_{I}$ be the bifurcation parameter.

Figure 2 shows a portion of a branch of periodic solutions. The quantity Norm, which is used as a convenient solution measure, is defined as

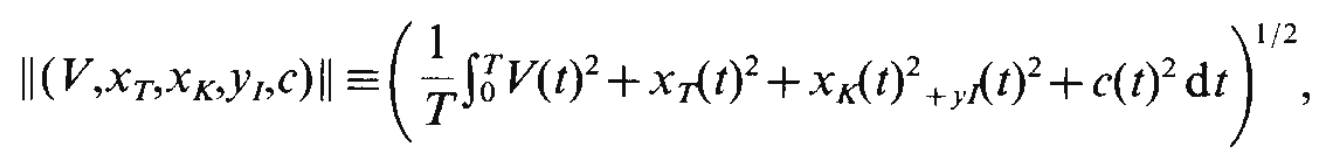

where $T$ is the period of the oscillation. Over subintervals of the displayed range of $g_{I}$, the qualitative behavior of the periodic orbits is characterized by the number of spikes. Between these intervals are very narrow regions where one spike is added as $g_{I}$ increases. Figures 3 and 4, with scaled-time variable $t$ as horizontal axis and with $V(t)$ as vertical axis, show representative bursting solutions carrying three and four spikes, respectively.

Figure 5 shows a blow-up of the bifurcation diagram around the second narrow region. To the left of this region, there is one spike; to the right, there are two spikes per period. Note that there is no branching process involved in the transition from one spike to two spikes. There are, however, cascades of period-doubling bifurcations. A 


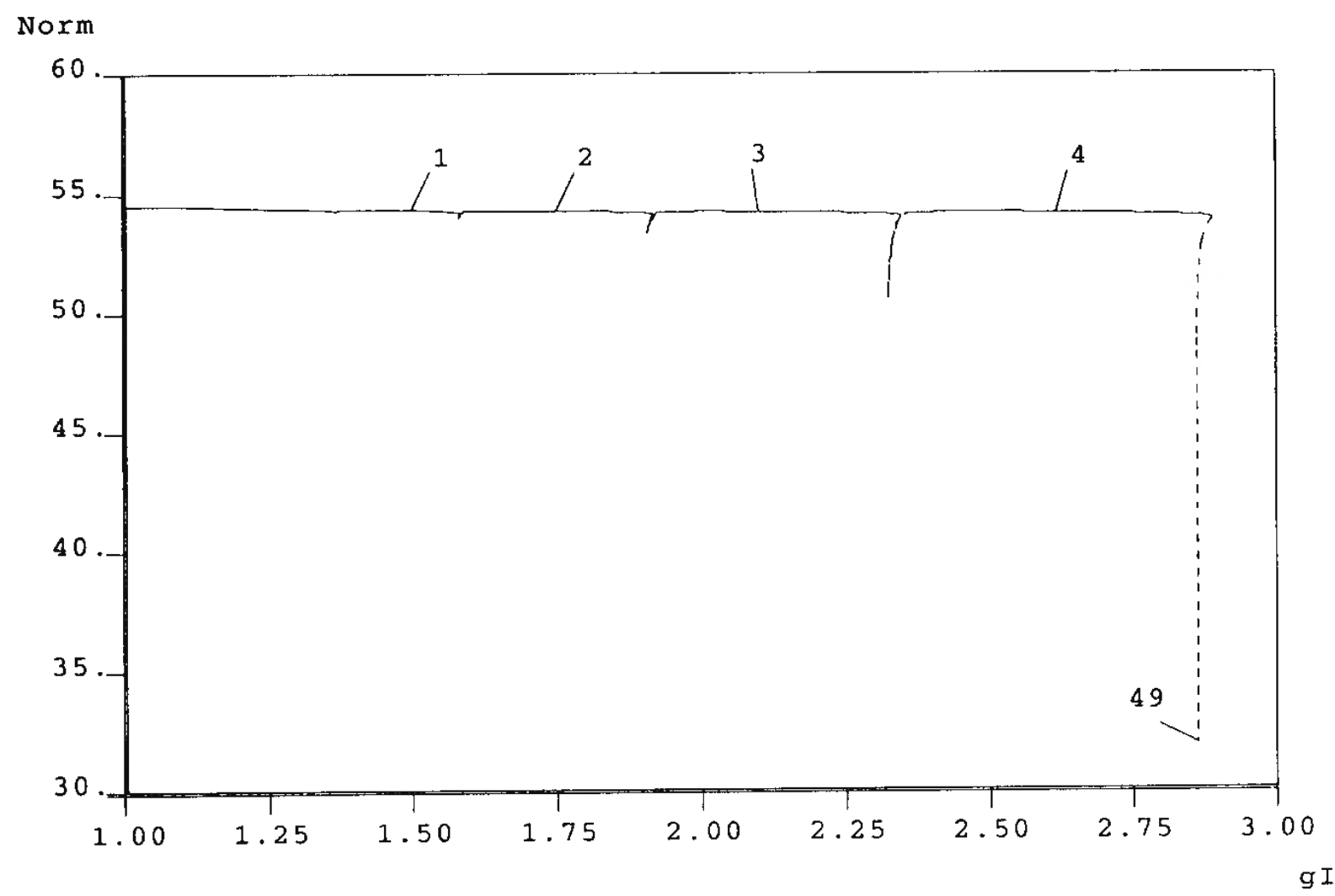

Fig. 2. Part of a branch of periodic solutions for Plant's model.

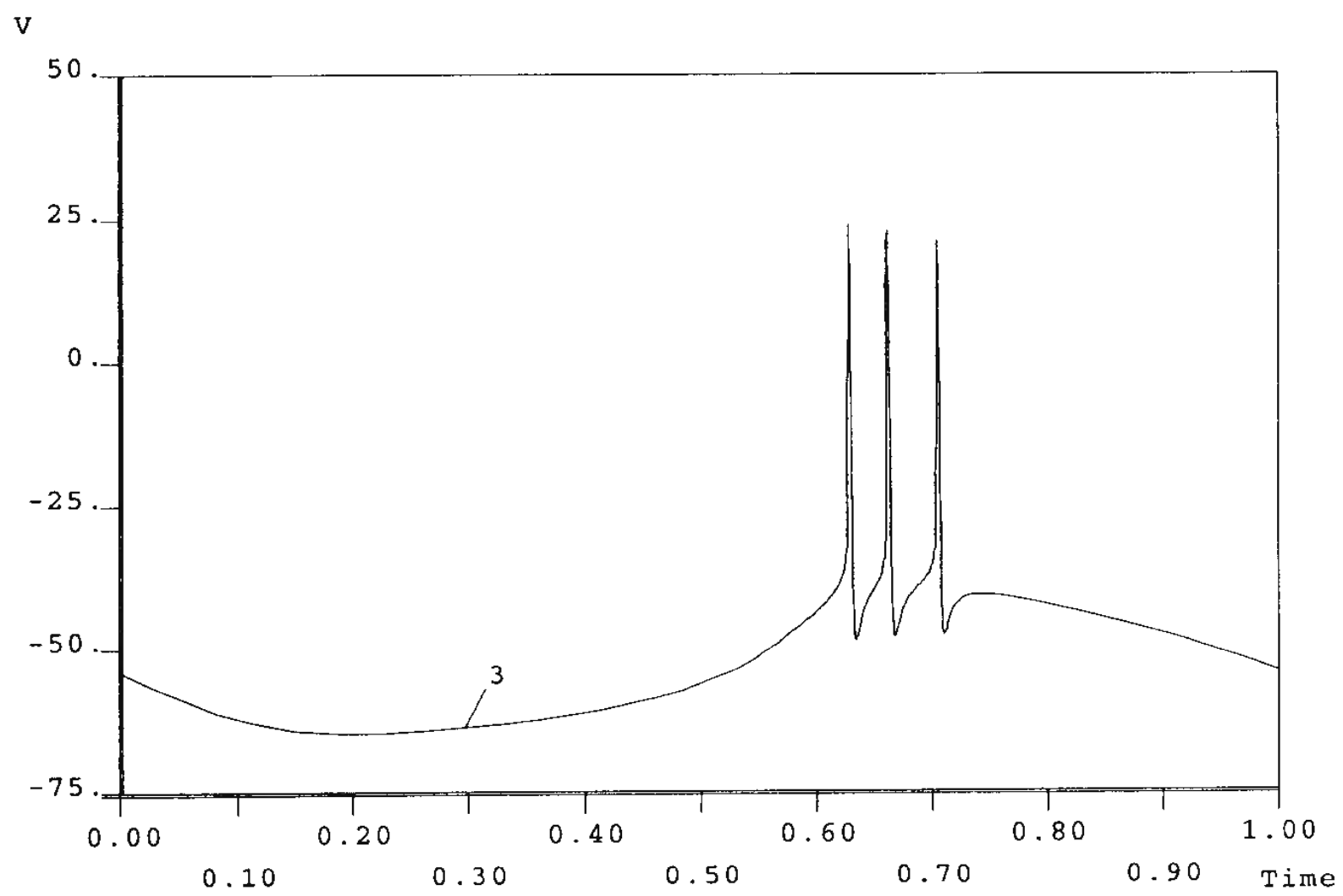

Fig. 3. $V(t)$ versus scaled time $t$ for solution 3 .

typical orbit on the first period-doubled branch is shown in Fig. 6. Note that the orbit alternates between one spike and two spikes.

The main branch terminates on the right in a homoclinic orbit. A very high period approximation to this homoclinic termination point is labeled 49 and shown in Fig. 7. It still carries four spikes, which are indistinguishable in the scaled-time plot due to the 


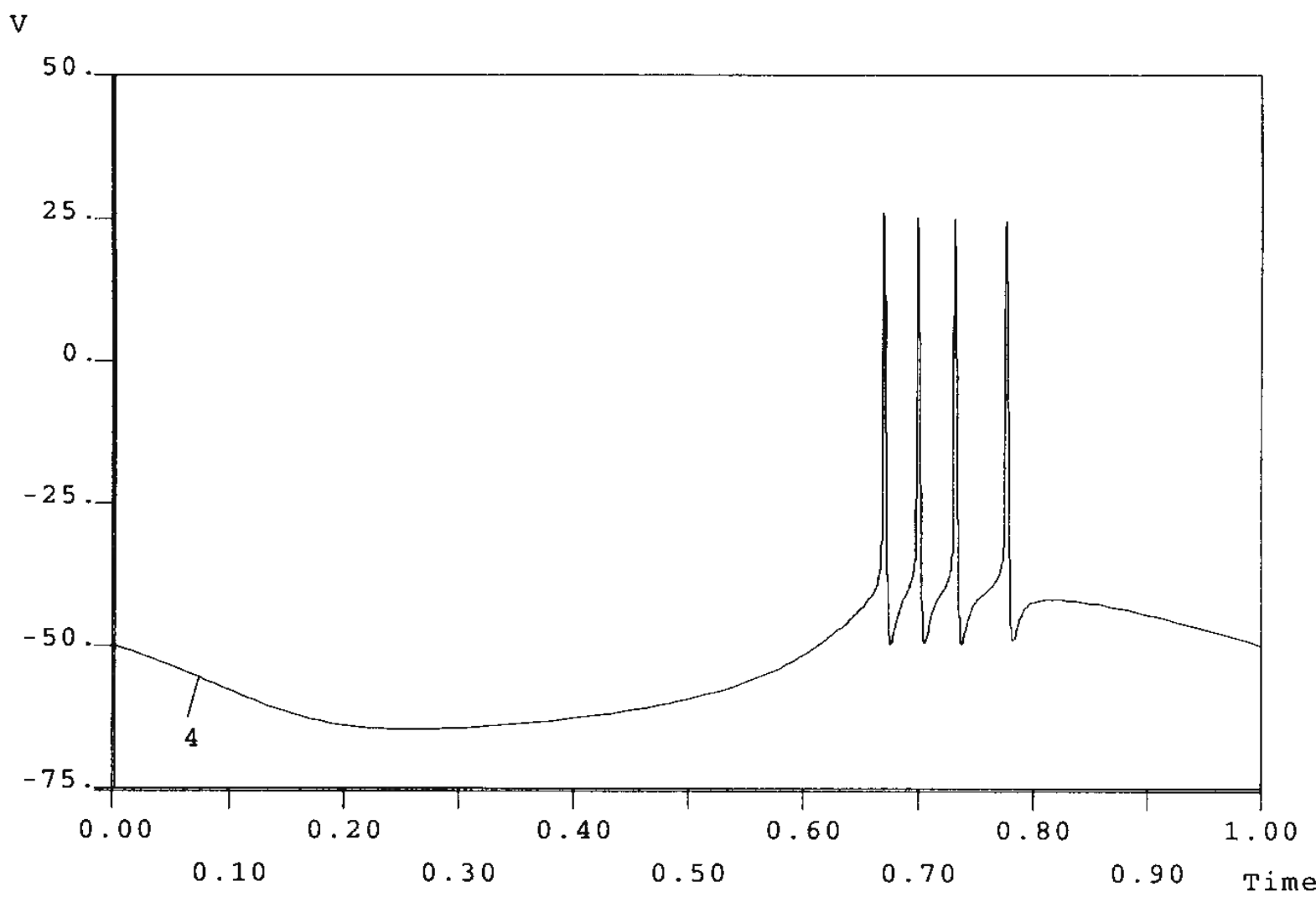

Fig. 4. $V(t)$ versus scaled time $t$ for solution 4 .

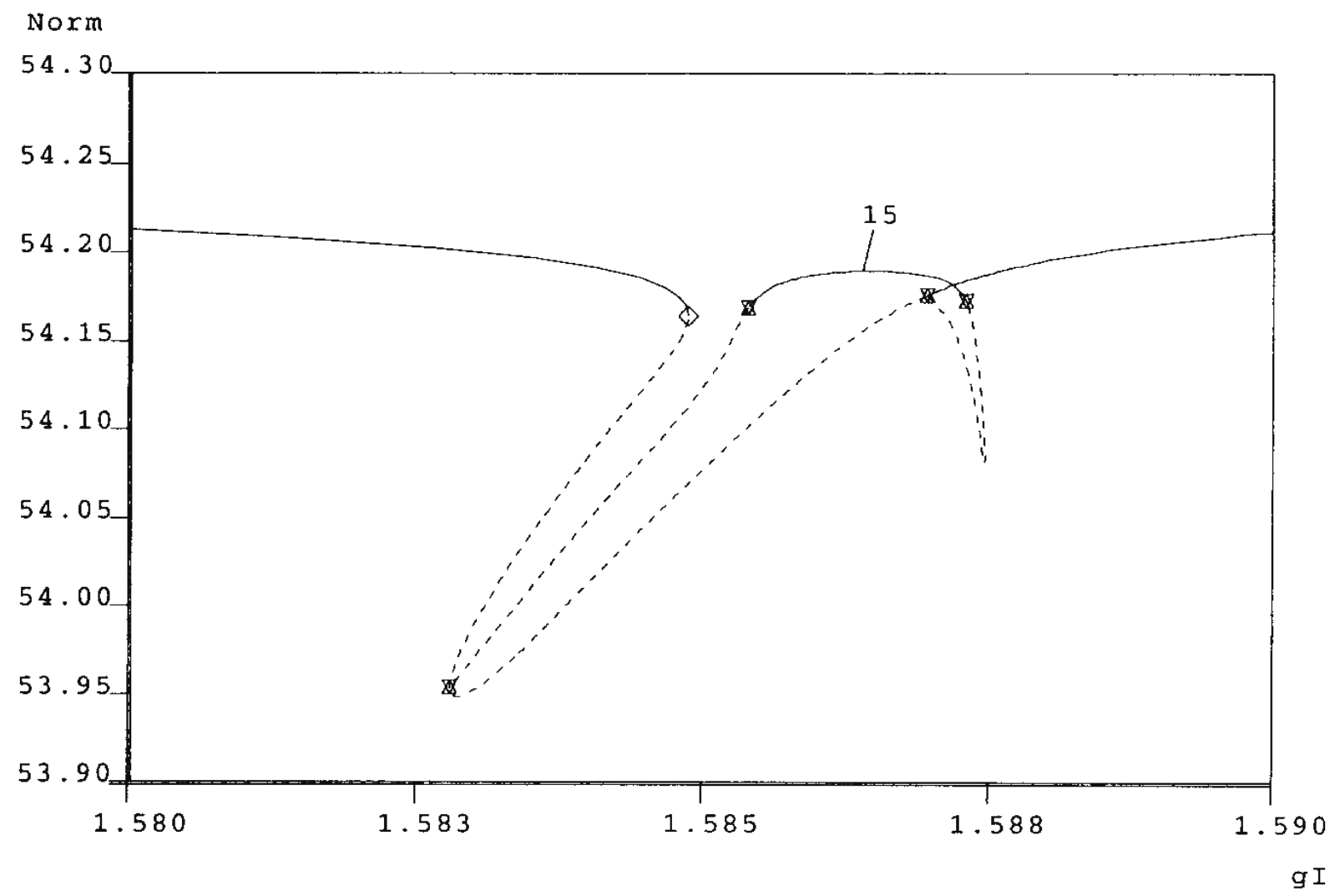

Fig. 5. Blow-up of the transition region between labels 1 and 2 .

extremely high value of the actual period. Nevertheless, a projected phase diagram (not shown) would clearly indicate four spikes. This orbit is another illustration of the accuracy and robustness of the computational methods in resolving near-singular problems. 


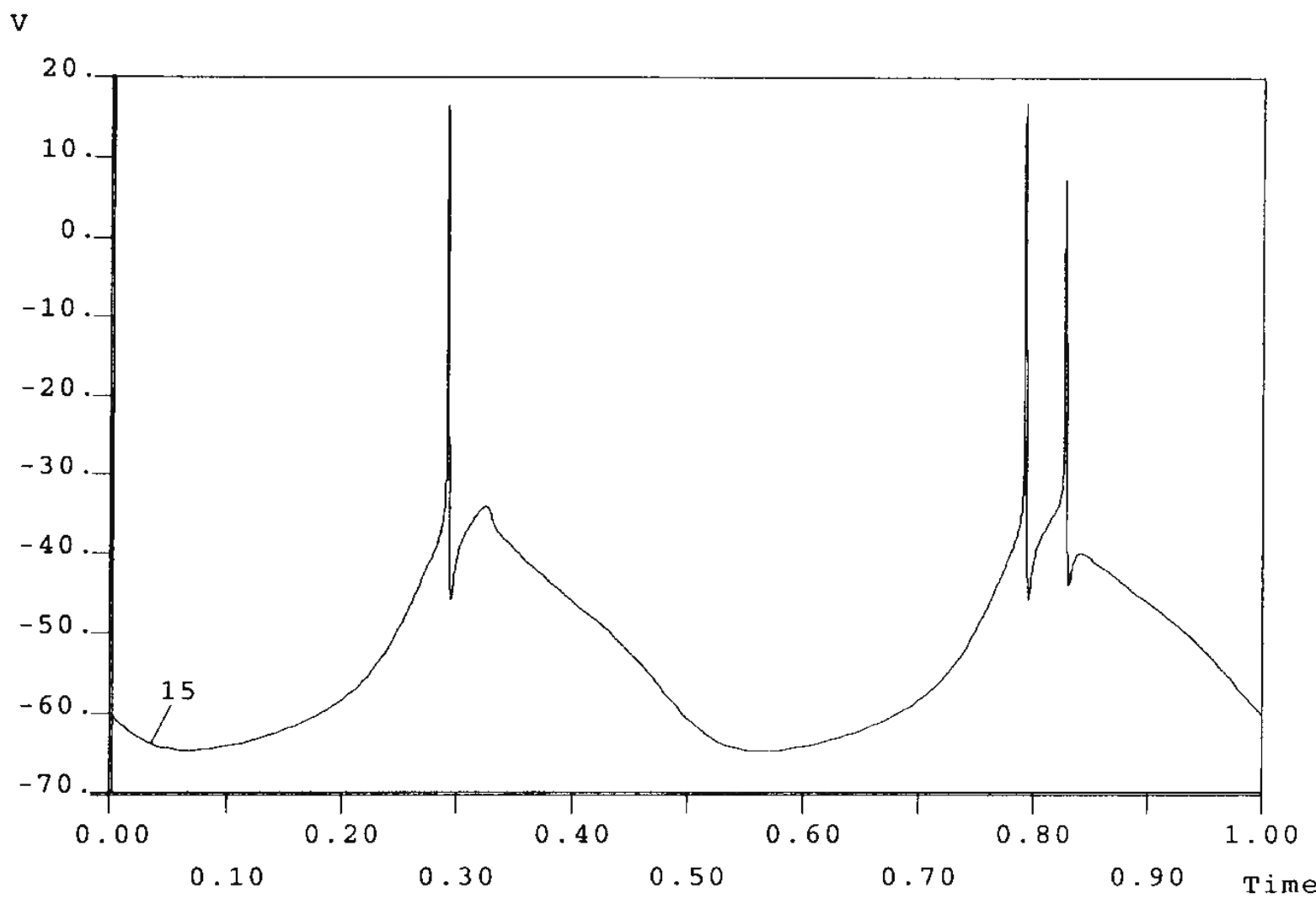

Fig. 6. $V(t)$ versus scaled time $t$ for solution 49 .

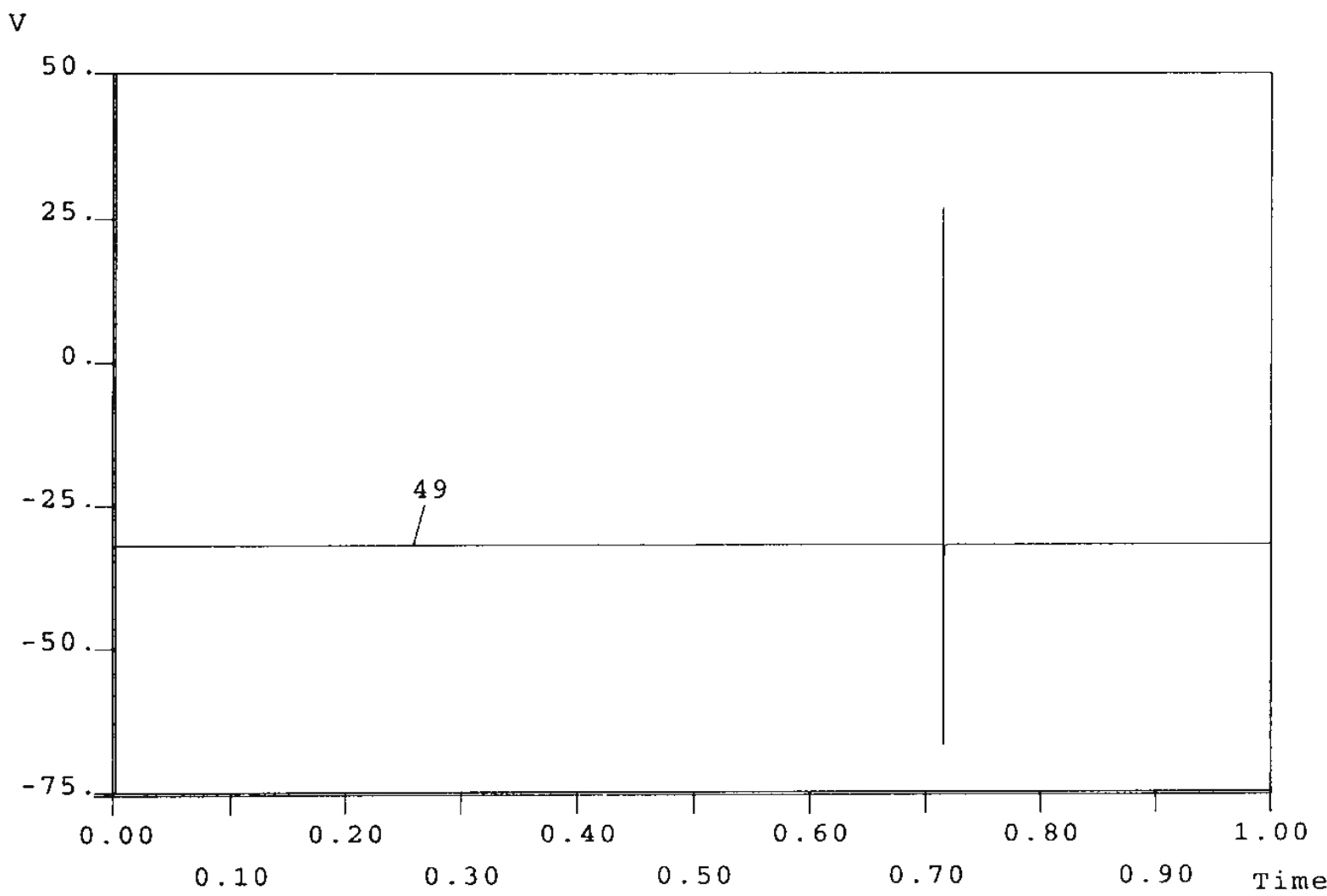

Fig. 7. $V(t)$ versus scaled time $t$ for solution 15 .

On the other hand, these calculations demand much computation time and, in extreme cases, become impossible due to ill-conditioning of the boundary value formulation. (Initial value calculations are entirely useless in resolving the transition regions and the homoclinic behavior.) Although the use of more numerically stable 
linear solvers may somewhat extend the computation domain, it is likely that the calculations described here approach the computability limits. Two time-scale geometric singular perturbation analysis is likely to be the remaining (analytical) tool. In fact, such analyses can be found, for example, in Rinzel (22) and Terman (23). See also Decroly and Goldbeter (24).

\subsection{Chua's circuit}

Chua's circuit is one of the simplest electronic devices to exhibit complex behavior. A reference related to the discussion here is Khibnik et al. (25). The equations modeling the circuit are

$$
\begin{aligned}
x^{\prime} & =\alpha[y-h(x)], \\
y^{\prime} & =x-y+z, \\
z^{\prime} & =-\beta y,
\end{aligned}
$$

where

$$
h(x)=m_{1} x+\frac{1}{2}\left(m_{0}-m_{1}\right)\{|x+1|-|x-1|\},
$$

and where we take $\beta=14.3, m_{0}=-1 / 7$ and $m_{1}=2 / 7$.

Note that $h(x)$ is not a smooth function, and hence the solution to the equations may have non-smooth derivatives. However, for the orthogonal collocation method to attain its optimal accuracy, it is necessary that the solution be sufficiently smooth. Moreover, the typical adaptive mesh selection strategies will fail if the solution, or one of its lower-order derivatives, has discontinuities. Although one can be very precise about the necessary degree of continuity, it is in practice reasonable to require that solutions be $C^{\infty}$. On the other hand, the adaptive mesh selection enables the resolution of "near-discontinuities", i.e. narrow regions in which the solution or some derivatives rapidly change. For these reasons, it is desirable that discontinuities be $C^{\infty}$ approximated. Thus we use the smooth approximation

$$
|x| \approx \frac{2 x}{\pi} \arctan (K x),
$$

which get better as $K \rightarrow \infty$. In the numerical calculations, we have used a very large value of $K$, namely $K=10^{6}$.

Figure 8 shows a period-doubling sequence and Fig. 9 displays the last computed orbit on the second period-doubling branch. All three branches terminate in Shilnikov homoclinic orbits and, due to symmetry, these orbits occur in pairs. The displayed orbit, which has period $T=2000$, is an approximation to one such homoclinic orbit. It is represented in Fig. 9 using $x$ and $x^{\prime}$ as coordinates, which makes the rapid but smooth change of $x^{\prime}$ near $x=-1$ clearly visible. The accuracy and adaptive mesh selection of the orthogonal collocation method enables the resolution of this nearsingularity, which occurs six times along the displayed orbit. Moreover, the perioddoubling bifurcations remain detectable along the computed solution branches. However, the continuation process and the Floquet multiplier computation become less robust as $K$ increases. 


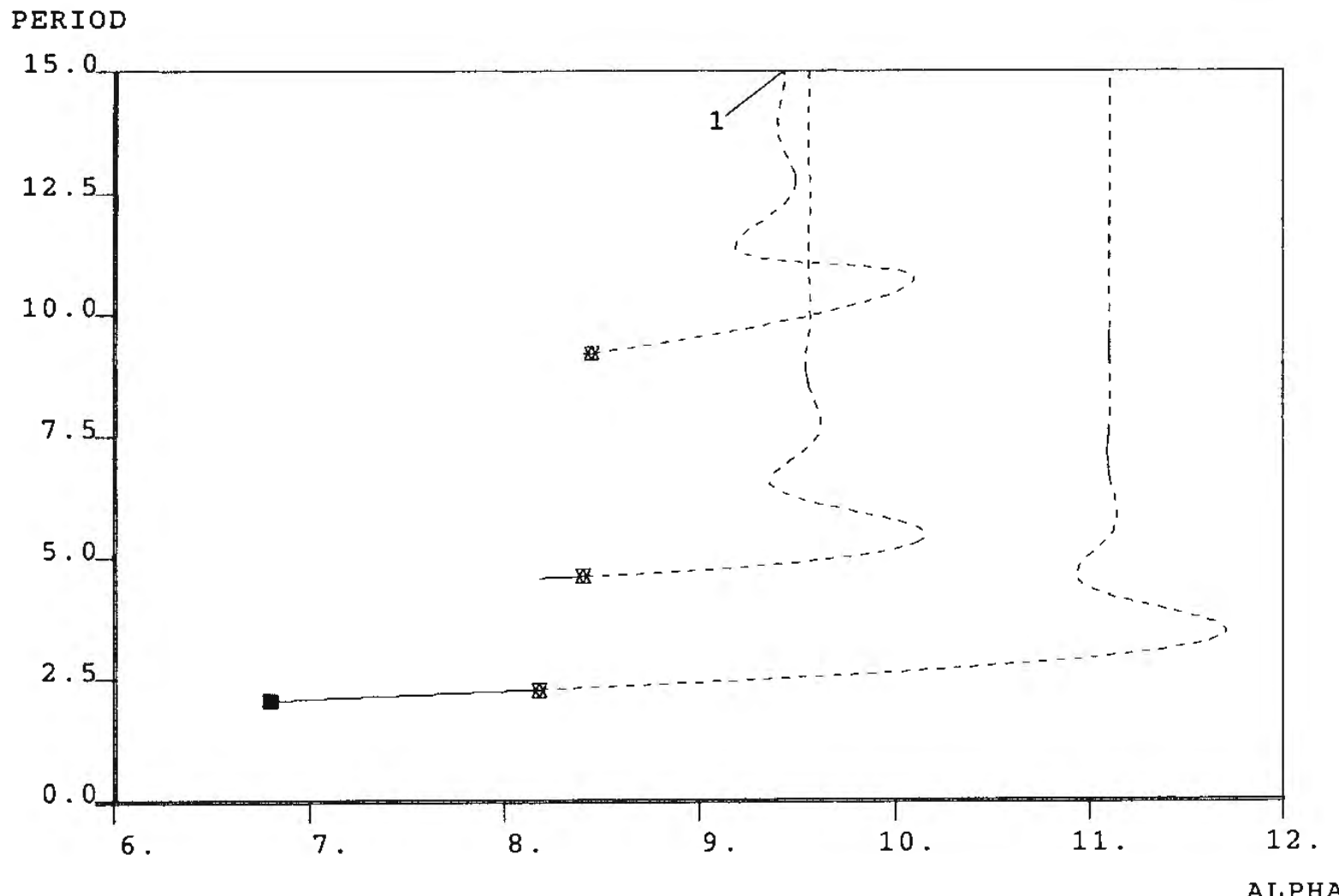

Fig. 8. A portion of the bifurcation diagram for Chua's circuit.

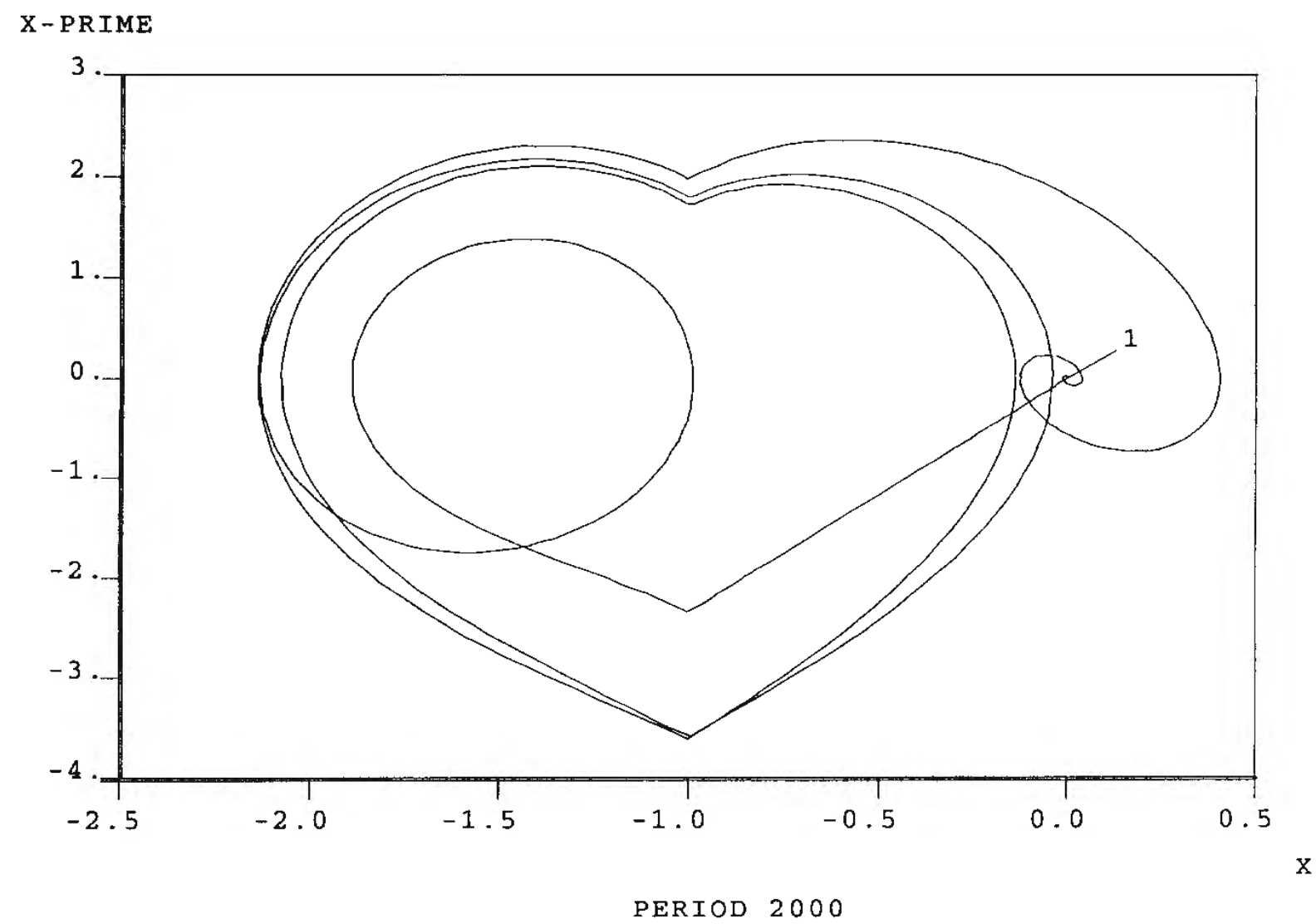

Fig. 9. An approximate homoclinic orbit (period 2000) in Chua's circuit.

\subsection{Coupled Josephson junctions}

Linear arrays of current biased Josephson junctions which are coupled through a shared load are used in various physical devices (26). Results on the general case of $n$ junctions can be found in Aronson et al. (27). Here we consider the case of two junctions 
treated in Aronson et al. (28). Let $\varphi_{j}$ be the phase difference across the gap in the $j$ th junction, for $j=1,2$. If the load is a capacitor then, after some algebra and appropriate normalization, the evolution of the $\varphi_{j}$ is governed by the equations

$$
\beta \varphi_{j}^{\prime \prime}+\varphi_{j}^{\prime}+\sin \varphi_{j}-\frac{3}{2(3+\beta)} \sum_{k=1}^{2}\left(\varphi_{k}^{\prime}+\sin \varphi_{k}\right)=\frac{\beta}{3+\beta} I, j=1,2,
$$

where $\beta$ is the capacitance of the individual junctions and $I$ is a bias current. Assume that $I \in(0,1)$, and let $a \equiv \arcsin I$ and $p \equiv \pi-\arcsin I$. Then there are four rest points for $\left(\varphi_{1}, \varphi_{1}^{\prime}, \varphi_{2}, \varphi_{2}^{\prime}\right)$, namely,

$$
\begin{aligned}
& \mathbf{3} \equiv(a, 0, p, 0), \quad \mathbf{2} \equiv(p, 0, p, 0), \\
& \mathbf{1} \equiv(a, 0, a, 0), \quad \mathbf{4} \equiv(p, 0, a, 0) .
\end{aligned}
$$

Here the rest points have been visually ordered as they appear in the projected $\left(\varphi_{1}, \varphi_{2}\right)$ phase plane; see Figs $10-13$ for examples. Rest point 1, which is a sink, is not shown in Figs $10-13$ as it is of no interest here. Rest point $\mathbf{2}$ is a hyperbolic point with 2 dimensional stable and unstable manifolds, and rest points 3 and 4 are hyperbolic points with 3-dimensional stable and 1-dimensional unstable manifolds. We use a subscript + to denote translation by $2 \pi$ in the first coordinate and a superscript ${ }^{+}$to denote translation by $2 \pi$ in the third coordinate. Thus, for example, $\mathbf{3}_{+} \equiv(a+2 \pi, 0, p, 0)$, $\mathbf{3}^{+} \equiv(a, 0, p+2 \pi, 0)$, and $\mathbf{3}_{+}^{+} \equiv(\mathrm{a}+2 \pi, 0, \mathrm{p}+2 \pi, 0)$.

A solution $\left\{\varphi_{1}(t), \varphi_{2}(t)\right\}$ is a rotation if there exists a minimal $T>0$ such that $\varphi_{j}(t+T)=\varphi_{j}(t)+2 \pi$, for $j=1,2$. An in-phase rotation is one for which $\varphi_{1} \equiv \varphi_{2}$; see Fig. 10 for an example. Although the physical interest is mostly in in-phase rotations, it is also of interest to classify other solution types. Numerical simulations, as well as

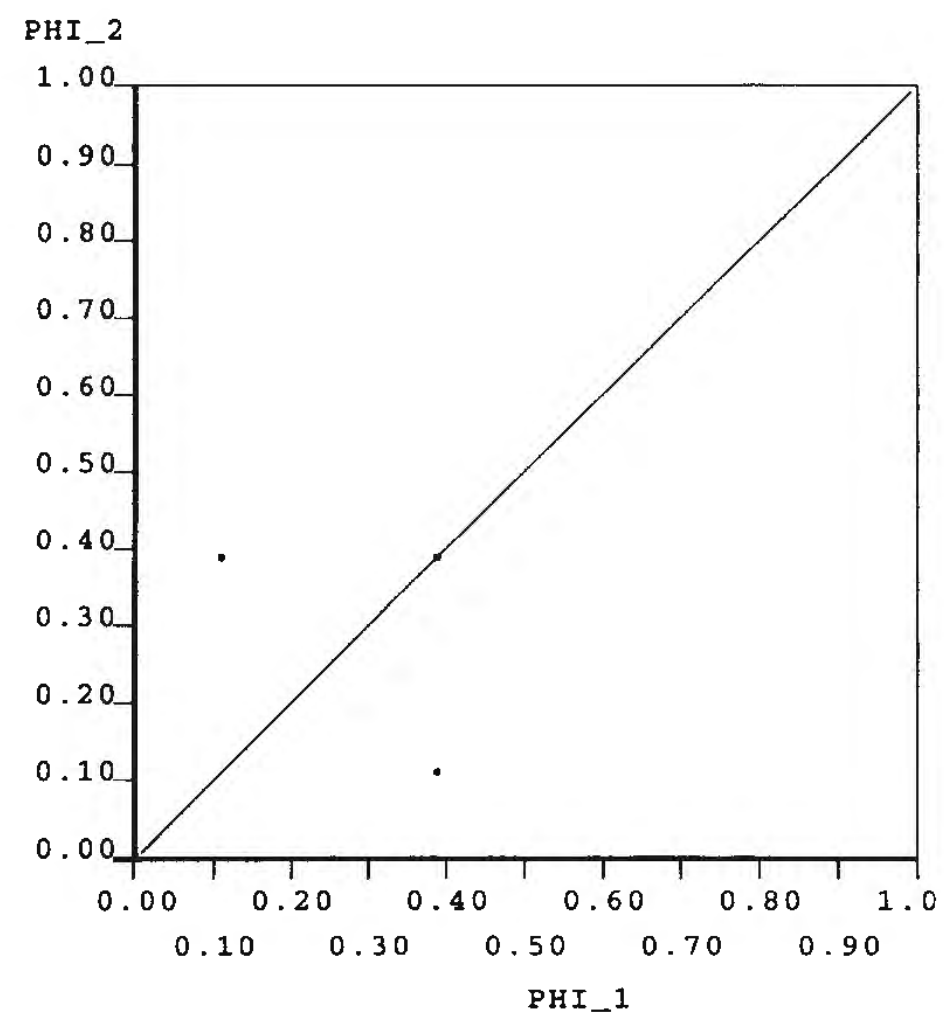

Fig. 10. Solution 1. 


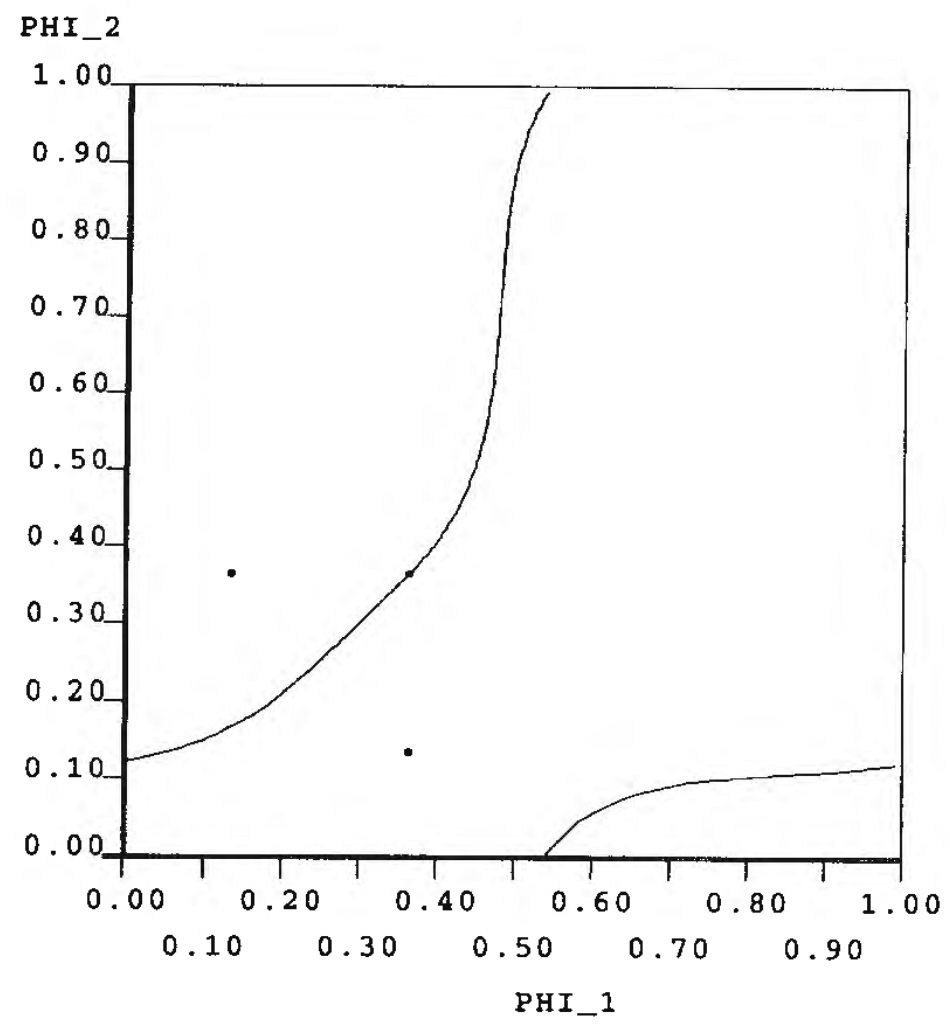

Fig. 11. Solution 2.

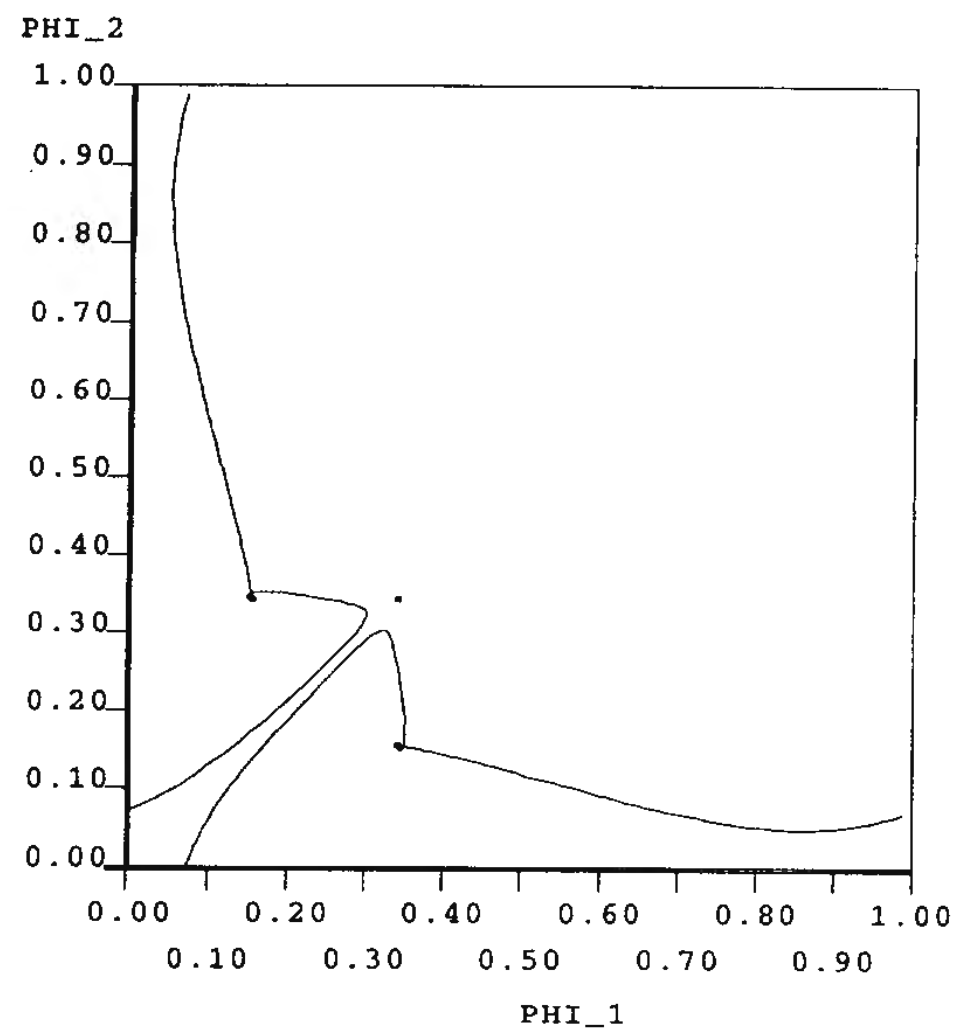

Fig. 12. Solution 4.

numerical continuation studies, show that the general rotation solution structure is very rich and difficult to describe.

In order to provide at least a skeleton of this structure, we have computed loci of rotations of infinite period, as they are generally in some way the limit of finite period rotations. In particular, all in-phase rotations originate from symmetric heteroclinic 


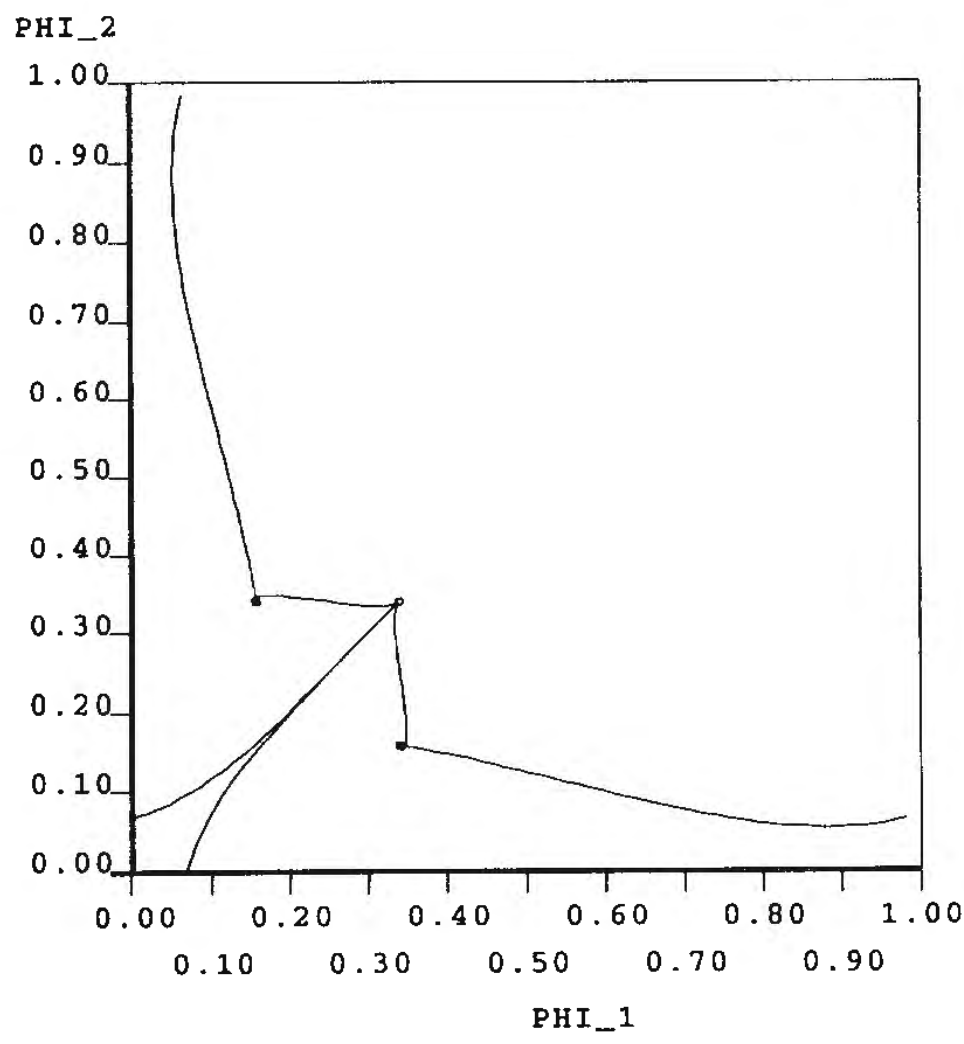

Fig. 13. Solution 8 .

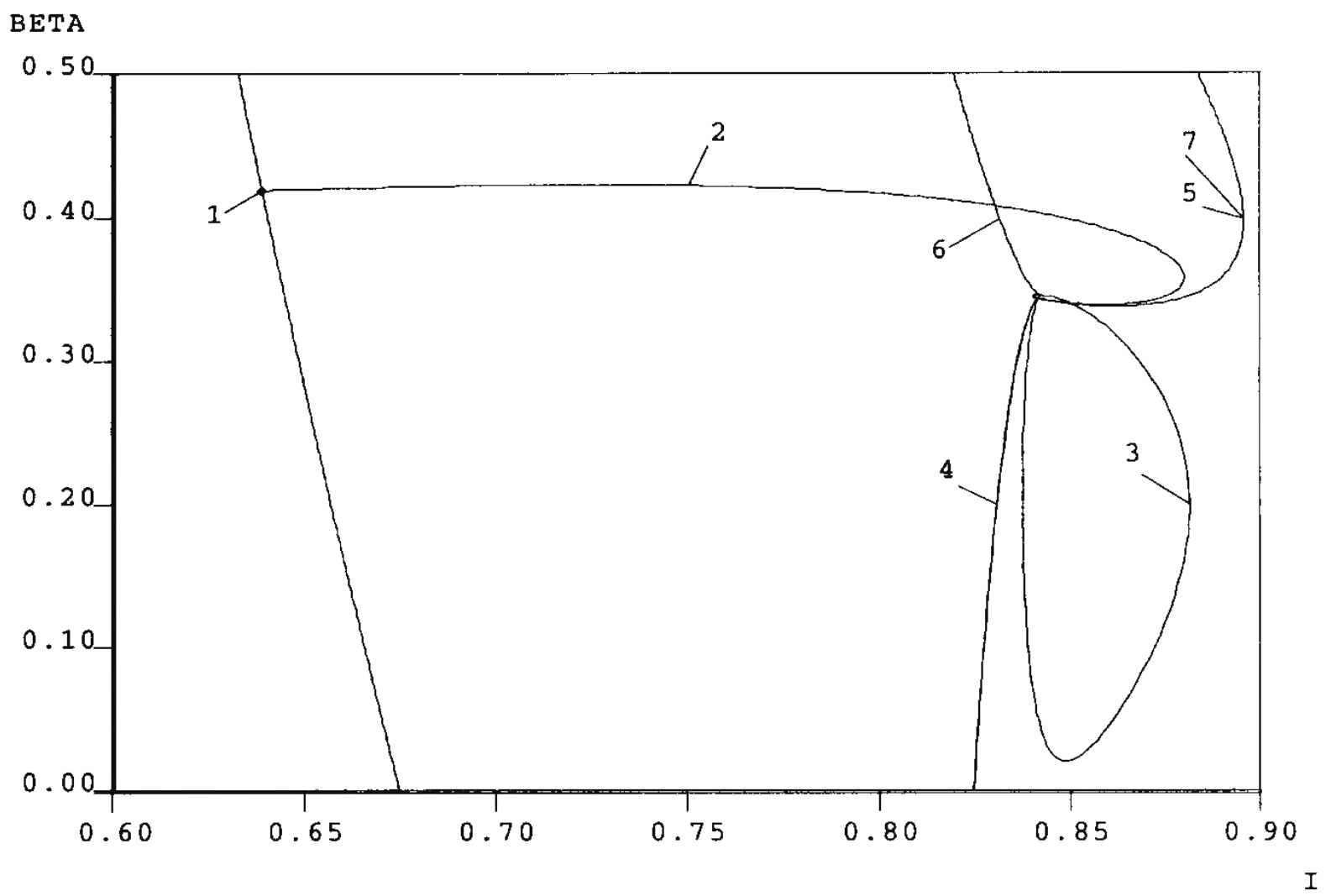

Fig. 14. Loci of connecting orbits for the Josephson junctions model.

connections between the rest points 2 and $2_{+}^{+}$. Since the stable and unstable manifolds of $\mathbf{2}$ and its translate $\mathbf{2}_{+}^{+}$are 2 -dimensional, this $\mathbf{2} \rightarrow \mathbf{2}_{+}^{+}$connection is codimension-1, and there is a curve $I=I(\beta)$ in parameter space, namely the leftmost curve in Fig. 14, along which these heteroclinic connections exist.

The existence of a codimension- 2 bifurcation point, at solution 1 in Fig. 14, on the 


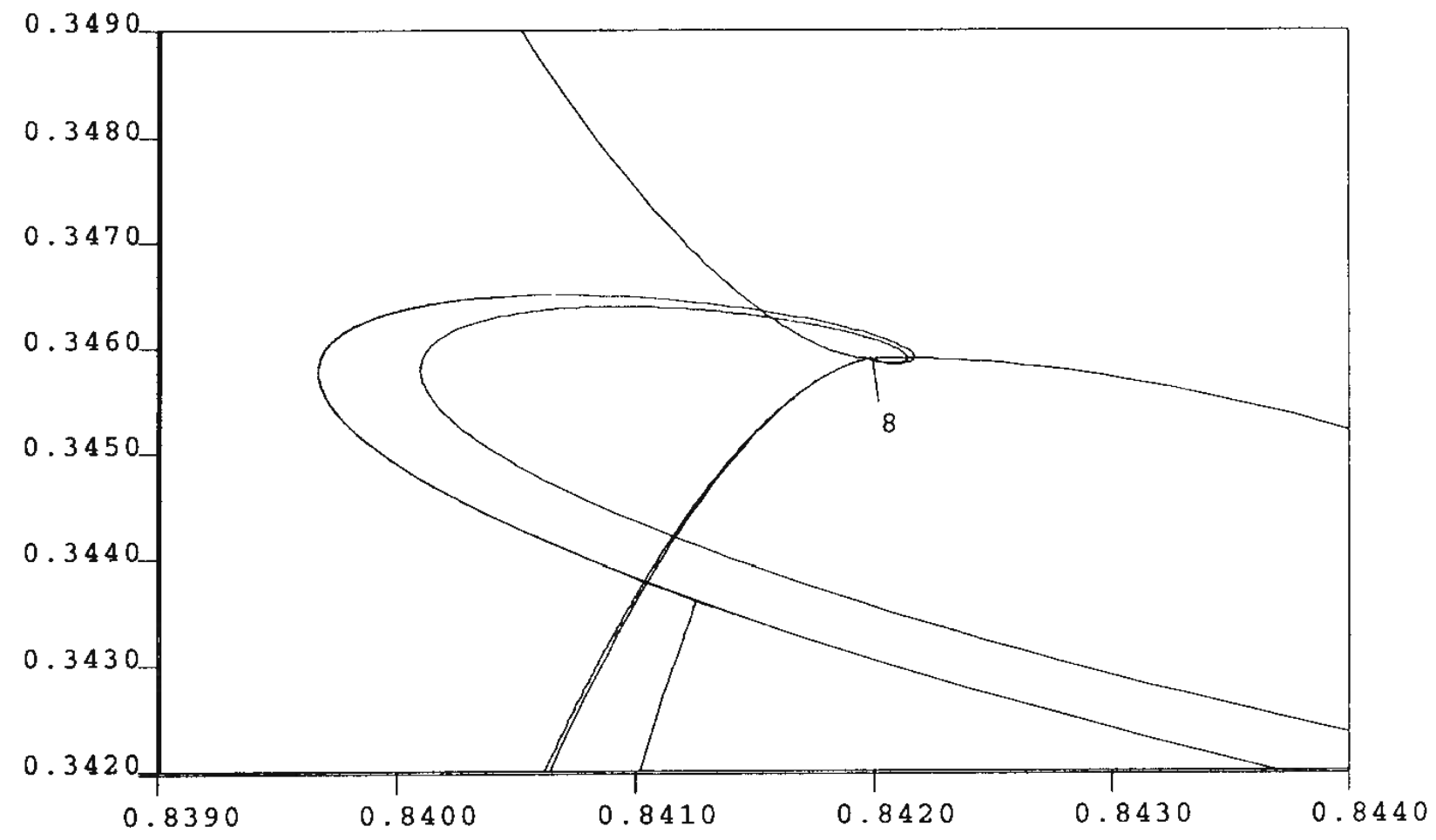

Fig. 15. A blow-up of a neighborhood of the codimension-2 point.

curve $I=I(\beta)$ is proved in Aronson et al. (27). This is a homoclinic twist bifurcation and there is a branch of asymmetric $\mathbf{2} \rightarrow \mathbf{2}_{+}^{+}$connections, called "bellows", that emanates from it. Figure 11 illustrates such a bellow. A different type of bellow, namely a $\mathbf{4} \rightarrow \mathbf{4}_{+}^{+}$connection, occurs along the curve which contains solution 3 .

Another solution type is the discrete rotating wave or "POM", for "pony on a merrygo-round". We find two branches of infinite-period POMs. One contains $\mathbf{4} \rightarrow \mathbf{3}_{+} \rightarrow \mathbf{4}_{+}^{+}$ connections; see, for example, solution 4 in Fig. 12. The other consists of $\mathbf{2}_{\rightarrow} \mathbf{2}_{+} \rightarrow 2_{+}^{+}$ connections; namely the branch carrying solution 5 . There are also two branches of socalled infinite period semi-rotors. One consists of $\mathbf{4} \rightarrow \mathbf{4}_{+}$connections (the branch carrying solution 6 ), while the other consists of $\mathbf{2} \rightarrow \mathbf{2}_{+}$connections (the branch with solution 7).

All branches, except the in-phase branch, appear to emanate from a single point $\mathbf{P}$, namely solution 8 in the enlarged diagram detail in Fig. 15. This is apparently a codimension- 2 point at which there is a $\mathbf{3} \rightarrow \mathbf{2}^{+}$, and by symmetry, a $\mathbf{4} \rightarrow \mathbf{2}_{+}$connection; see Fig. 13. Generically, these connections are codimension-2, since the unstable manifolds of $\mathbf{3}$ and $\mathbf{4}$ are both one-dimensional, while the stable manifold of $\mathbf{2}$ is 2 -dimensional.

Unfolding results in Aronson et al. (28) imply the existence of a countably infinite number of branches of $\mathbf{2} \rightarrow \mathbf{2}_{+}^{+}$connections near $\mathbf{P}$ that spiral into $\mathbf{P}$, and a countably infinite number of branches of $\mathbf{4} \rightarrow \mathbf{4}_{+}^{+}$connections near $\mathbf{P}$ that terminate in $\mathbf{P}$, but that do not spiral. The numerical location of such additional branches has been very difficult, especially the additional spiralling branches. In fact, we were at first unable to obtain numerical evidence of these theoretically predicted solutions. It was not until the search was extended to very large period that we were able to detect another such $\mathbf{4} \rightarrow \mathbf{4}_{+}^{+}$ connection. (We mostly used the high-period "periodic" approximation, rather than the asymptotic boundary conditions discussed earlier.) 


\section{Software development directions}

There is a need for further refinement of existing continuation algorithms and software for bifurcation analysis, and there is a need for their extension to new classes of problem. Below, a brief overview is presented of some problems and applications that especially merit further attention. The main categories considered are algorithms and software for singularities and manifolds, for some special problems and for largescale problems.

\subsection{Interactive software}

There is much current interest in computer user-interfaces and communication tools. Although it is important that algorithms be made as accessible as possible, there is a danger of creating a virtual world of empty shells. The emphasis should therefore remain on the algorithmic development within simple structures that allow updating, extension, and comparison of algorithms. In dynamical systems, there has been some progress in this direction. One notable achievement is the DsTool software (29), which includes some continuation capabilities. Another ongoing project, CONTENT (30), based on the earlier LOCBIF $(\mathbf{2 5}, \mathbf{3 1})$ and on AUTO (32), is primarily continuation oriented.

\subsection{Singularities}

5.2.1. Problems with symmetry. Multiple bifurcations do not occur in generic systems with one parameter. In fact, even simple bifurcation does not arise. Thus, one could concentrate on codimension-1 bifurcations, namely folds, Hopf bifurcations, perioddoubling and torus bifurcations, and homoclinic orbits, use extended systems to follow these in two-parameters, detect codimension-2 bifurcations, etc. Ironically, most systems that arise in applications are not generic. However, one can introduce suitable unfolding parameters, much like the variable $\mu$ in Section 2.2, to regularize the computations. The same can be done to deal with Hamiltonian systems (for an example, see Doedel et al. (33)). Nevertheless, it is often useful to deal directly with non-generic systems, such as symmetric systems, as one may want to exploit symmetries to reduce the computational complexity. The software SYMCON (34) is an important development in this direction.

5.2.2. Numerical linear algebra. In a typical numerical continuation calculation, most computer time is taken by the setting up and the numerical solution of the linear systems that arise in Newton's method. As mentioned above, if the problem has symmetries then the cost of the linear algebra can often be reduced. More generally, if the Jacobian matrices have a special structure then this can often be exploited in the linear algebra. An example that covers the two-parameter continuation of folds and higher-order singularities was given in Section 2.3. As important as efficiency is the numerical stability of the solution algorithms, given the nature of limiting problems encountered in continuation calculations. Further work on algorithms, and their inclusion in numerical bifurcation software, remains important. For recent work, see Govaerts (10).

5.2.3. Homoclinic bifurcations. In Section 3.3, we briefly described a scheme for computing branches of connecting orbits. The method applies to both homoclinic and 
heteroclinic orbits. Connecting orbits are important in the study of wave solutions to nonlinear diffusive systems. They also serve as "organizing curves" ("skeletons") from which other solution behaviors can be inferred, as in the Josephson junction example in Section 4.4.

Several unfolding results for singular homoclinic orbits have appeared in the literature; see Kuznetsov (35) for an introduction. Numerical determination and continuation of codimension-2 homoclinic bifurcations has rapidly advanced in recent years. See, for example, Champneys and Kuznetsov (36) and references therein. There is prototype software for the detection of 16 different codimension-2 bifurcations (37) which has been incorporated in AUTO (19).

5.2.4. Optimization. There is much current interest in controlling chaos, as evidenced by the many articles on the subject. Continuation techniques have played little or no role in these investigations. However, continuation techniques can be very effective in the stabilization of a desired solution type. One approach is the successive continuation technique, a strategy for the location and continuation of quadratic singular points of successively increasing codimension $(3, \mathbf{4})$. It applies in particular to the continuation of quadratic extrema of an objective functional on a solution manifold. Although, in practice, it is not possible to guarantee that a globally optimal solution will be reached, the technique does determine the fate of a given optimal solution as an additional parameter varies. This provides geometric insight, especially if there are few control parameters. Applications include, for example, the search for solutions with some desired feature, the stabilization of periodic orbits by controlling critical Floquet multipliers, and the optimal placement or annihilation of an instability source (e.g. a Hopf bifurcation). Although the procedure is more time consuming than standard optimization techniques, its Jacobian matrices have the special structure considered in Section 2.3. Efficient implementation in a continuation package remains an unaccomplished goal.

\subsection{Manifolds}

5.3.1. Implicitly defined $2 D$ manifolds. The calculation of solution curves and the continuation of curves of singular points of successively increasing codimension give much insight into the solution structure of nonlinear equations. In some applications, however, it is useful to calculate two-dimensional, or even higher-dimensional solution manifolds or singularity manifolds. See Allgower and Georg (5) for a survey of some basic numerical techniques. A global scheme was developed by Henderson (38), and a current software project (Pisces) for computing manifolds and their singularities is in progress (39).

5.3.2. Invariant manifolds. An important manifold type is the invariant manifold in dynamical systems. Simple examples are the stable and unstable manifolds of hyperbolic fixed points. Software for these has been developed by, for example, Kevrekidis and Jolly (40) and Nusse and Yorke (41). Less work has been done on the computationally more difficult case of the stable and unstable invariant manifolds of a periodic orbit. This problem can be reduced, via the Poincaré map, to computing the corresponding manifolds of a fixed point. This suggests shooting, but its poor performance for unstable orbits makes a direct approach preferable.

Particularly challenging is the continuation of an invariant torus, a generalization of 
the periodic orbit. Although a general persistence theory has been known for some time (42), feasible computational schemes have only recently emerged; see, for example, Lorenz (43) and references therein. The special case of an invariant "circle" has been treated more extensively; see, for example, Broer et al. (44) and references therein.

\subsection{Special ODEs}

5.4.1. Delay differential equations. Relatively little work has been done on the development of numerical continuation and bifurcation software for delay differential equations and, more generally, for functional differential equations. There is a software package XPPAUT (45) that can do simulation calculations and some numerical continuation, but it does not include, for example, the continuation of periodic solutions. Indeed, the latter problem is inherently infinite-dimensional. Certain numerical schemes were developed and tested many years ago $(\mathbf{4 6}, \mathbf{4 7})$, but no generally distributed continuation software exists for this purpose. Promising recent work has been done by Cometto et al. (48) and Luzyanina et al. (49).

5.4.2. Differential algebraic equations. Differential algebraic equations (DAEs) have been of some interest in numerical analysis in recent years. For the case of importance here, namely ODE boundary value problems (Section 3), an orthogonal collocation code COLDAE has been developed by Ascher and Spiteri (50). However, a general continuation and bifurcation code for DAEs does not exist.

5.4.3. Systems with discontinuities. As illustrated in Section 4.3, one can approximate discontinuous systems by smooth systems to which standard discretization and continuation applies. However, the "near-discontinuities", which can be resolved by a good adaptive mesh, require a very accurate discretization, worsen the conditioning of the linear solvers, and reduce the continuation stepsize. In extreme cases, this will limit the effectivenes of a numerical analysis, and one must deal directly with the discontinuities. One approach is to treat the location of discontinuities as unknowns and, after appropriate transformations of the independent variable, obtain an enlarged system on the unit interval with scalar unknowns corresponding to the unknown interfaces. However, the number of interfaces may be unknown beforehand and change during continuation, causing implementation difficulties. There are also fundamental theoretical difficulties in the bifurcation study of such systems.

\subsection{Large problems}

5.5.1. ODEs. As already mentioned, there is a need for further refinement of the linear algebra algorithms in numerical continuation. This is especially true for large systems. Optimal mesh selection may also need re-evaluation. It is not clear, whether the current selection criteria are indeed optimal near, for example, homoclinic orbits. For very large ODE systems, there is a need for easy-to-install and easy-to-use parallel software versions. A prototype implementation is described in Wang and Doedel (18).

5.5.2. PDEs. The greatest challenges lie, not surprisingly, in the development of numerical continuation and bifurcation software for PDEs. There is such a package for scalar nonlinear elliptic PDEs on general domains in $R^{2}$, namely PLTMG (51). It includes continuation and some branch switching capabilities, and has been very successful in various applications. The underlying solution technique is that of multigrid, which is very fast, but whose performance typically degrades when the problem 
becomes difficult. However, such iterative techniques are a necessity for large scale PDE problems in $R^{3}$ and higher dimensions. Good results have also been obtained with stabilized simple iteration schemes for computing stationary PDE solutions "with mostly stable modes"; see, for example, Schroff and Keller (52).

There remains a need for general bifurcation software for systems of elliptic PDE, subject to general boundary conditions and integral constraints. For the case of such systems on simple domains in $R^{2}$, the generalization of the collocation method of Section 3.5 carries some promise. To become comparable to current ODE bifurcation software, it will be necessary to use adaptive meshes. In this case, the direct solution of the linear systems arising in Newton's method remains feasible, so that a high degree of robustness is possible. Interesting applications include, for example, 2D Navier Stokes problems. Extension to mixed PDE/ODE systems would be desirable.

\section{Discussion}

The effective use of numerical continuation for the bifurcation analysis of nonlinear equations demands a certain level of knowledge of bifurcation theory, of numerics and, of course, of the intended application area. In the case of dynamical systems, this is in contrast with simple simulations, which will give results, whether correct or not, under almost any conditions. In a numerical analysis, one must understand and confront the fundamental reasons of a qualitative change of behavior, have some understanding of their possible impact on the numerics, and be able to interpret the results in the context of the application. Computational difficulties encountered, or even the results themselves, may not be understood or appreciated, without some knowledge of the fundamentals of bifurcation theory and numerical analysis. It seems desirable, therefore, that applied dynamical systems in curricula have a firm theoretical basis, in terms of bifurcation theory, and a sound algorithmic basis, in terms of numerical analysis.

As the examples in the previous sections have illustrated, there are practical computability limits, in terms of computer time, necessary precision, and complexity of the solution structure. Often one is interested in a limiting behavior, for which continuation is a suitable tool, but which leads to ever increasing accuracy requirements and numerical ill-conditioning, hence requiring ever increasing machine precision and computer time. Thus, the significance of computed results cannot be fully understood without recourse to analytical methods, such as singularity theory, perturbation analysis, or global geometric methods.

However, within the limits of computability, the advantages of a numerical bifurcation analysis can be numerous. Transitions from stable to unstable behavior can often be pinpointed, while phenomena that underlie transitions can frequently be determined, whether physically observable (i.e. asymptotically stable) or not. The numerical results often provide a much simplified representation of the fundamental features of solution behavior, a "skeleton", from which other behaviors can, to some extent, be deduced. Sometimes the numerical results may even suggest new methods or theories, as was the case, for example, for the coupled Josephson junctions model in Section 4.4. For dynamical systems, continuation greatly reduces the dimensionality of the computation space, as the results are not functions of initial conditions. Indeed, a 
carefully computed bifurcation diagram often carries more fundamental information than an entire picture book of simulation results can provide.

\section{References}

(1) Rheinboldt, W. C., Numerical Analysis of Parametrized Nonlinear Equations. University of Arkansas Lecture Notes in the Mathematical Sciences, Wiley-Interscience, 1986.

(2) Seydel, R., From Equilibrium to Chaos. Practical Bifurcation and Stability Analysis, 2nd edn. Springer, New York, 1994.

(3) Doedel, E. J., Keller, H. B. and Kernévez J. P., Numerical analysis and control of bifurcation problems: (I) Bifurcation in finite dimensions. Internat. J. Bifur. Chaos, 1991, 1, 493-520.

(4) Doedel, E. J., Keller, H. B. and Kernévez J. P., Numerical analysis and control of bifurcation problems: (II) Bifurcation in infinite dimensions. Internat. J. Bifur. Chaos, 1991, 1, 745772.

(5) Allgower, E. L. and Georg, K., Numerical path following. In Handbook of Numerical Analysis, Vol. 5, ed. P. G. Ciarlet and J. L. Lions. North Holland Publishing, 1996.

(6) H. B. Keller, Numerical solution of bifurcation and nonlinear eigenvalue problems. In Applications of Bifurcation Theory, ed. P. H. Rabinowitz. Academic Press, 1977, pp. 359384.

(7) Kearfott, R. B., An interval step control for continuation methods. SIAM J. Numer. Anal., 1994, 31(3), 892-914.

(8) Chow, S.-N. and Hale, J., Methods of Bifurcation Theory. Springer, 1982.

(9) Wittenberg and Holmes, F., Princeton University, 1996.

(10) Govaerts, W., Computation of singularities in large nonlinear systems. SIAM J. Numer. Anal., 1997, 34(3), 867-880.

(11) Isaacson, E. and Keller, H. B., Analysis of Numerical Methods. Wiley, 1966.

(12) Lentini, M. and Keller H. B., Boundary value problems over semi-infinite intervals and their numerical solution. SIAM J. Numer. Anal., 1980, 17, 557-604.

(13) Beyn, W.-J., The numerical computation of connecting orbits in dynamical systems. IMA J. Numer. Anal., 1990, 9, 379-405.

(14) Friedman, M. J. and Doedel E. J., Numerical computation and continuation of invariant manifolds connecting fixed points. SIAM J. Numer. Anal., 1991, 28, 789-808.

(15) Ascher, U. M., Matthei j, R. M. M. and Russell, R. D., Numerical Solution of Boundary Value Problems for Ordinary Differential Equations. Prentice-Hall, 1988.

(16) de Boor, C. and Swartz B., Collocation at Gaussian points. SIAM J. Numer. Anal., 1973, 10, 582-606.

(17) Fairgrieve, T. F. and Jepson A. D., O.K. Floquet multipliers. SIAM J. Numer. Anal., 1991, 28(5), 1446-1462.

(18) Wang, X.-J. and Doedel, E.J., AUTO94P: An experimental parallel version of AUTO. CRPC-95-3, Center for Research on Parallel Computing, California Institute of Technology, Pasadena CA 91125, 1995.

(19) Doedel, E. J., On the construction of discretizations of elliptic partial differential equations, J. Difference Equations and Applications, to appear.

(20) Lorenz, J., Nonlinear boundary value problems with turning points and properties of difference schemes. In Singular Perturbation Theory and Applications, ed. W. Eckhaus and E. M. de Jager. Lecture Notes in Mathematics Vol. 942, Springer, 1982.

(21) Plant, R. E., Bifurcation and resonance in a model for bursting nerve cells. J. Math. Biol., 1981, 11, 15-32.

(22) Rinzel, J., Bursting oscillations in an excitable membrane model. In Ordinary and Partial Differential Equations, ed. B. D. Sleeman and R. J. Jarvis. Springer, 1985, pp. 304-316. 
(23) Terman, D., The transition from bursting to continuous spiking in excitable membrane models. J. Nonlinear Sci., 1992, 2, 135-182.

(24) Decroly, O. and Goldbeter A., From simple to complex oscillatory behaviour: Analysis of bursting in a multiply regulated biochemical system. J. Theoret. Biol., 1987, 124, 219 250 .

(25) Khibnik, A. I., Kuznetsov, Yu. A., Levitin, V. V. and Nikolaev E. N., Continuation techniques and interactive software for bifurcation analysis of ODE's and iterated maps. Phys. D, 1993, 62, 360-371.

(26) Hadley, P., Beasley, M. R. and Wiesenfeld K., Phase locking of Josephson-junction series arrays. Phys. Rev. B, 1988, 38, 8712-8719.

(27) Aronson, D. G., Golubitsky, M. and Krupa M., Coupled arrays of Josephson junctions and bifurcations of maps with $S_{N}$ symmetry. Nonlinearity, 1991, 4, 861-902.

(28) Aronson, D. G., Doedel, E. J. and Terman, D. H., A codimension-two point associated with coupled Josephson junctions, to appear in Nonlinearity.

(29) Back, A., Guckenheimer, J., Myers, M. R., Wicklin, F. J. and Worfolk P. A., DsTool: Computer assisted exploration of dynamical systems. Notices Amer. Math. Soc., 1992, 39(4), 303-309.

(30) Kuznetsov, Y. A. and Levitin, V. V., CONTENT, a multiplatform continuation environment. Technical report, CWI Amsterdam, 1996.

(31) Khibnik, A. I., Roose, D. and Chua L. O., On periodic orbits and homoclinic bifurcations in Chua's circuit with a smooth nonlinearity. Internat. J. Bifur. Chaos, 1993, 3(2), 363384.

(32) Doedel, E. J., Champneys, A. R., Fairgrieve, T. F., Kuznetsov, Y. A., Sandstede, B. and Wang, X.-J., AUTO97: Continuation and bifurcation software for ordinary differential equations. Department of Computer Science, Concordia University, Montreal, Canada, 1997 (Available by FTP from ftp.cs.concordia.ca. in directory pub/doedel/auto).

(33) Doedel, E. J., Aronson, D. G. and Othmer H. G., The dynamics of coupled current-biased Josephson junctions II. Internat. J. Bifur. Chaos, 1991, 1(1), 51-66.

(34) Gatermann, K. and Hohmann A., Symbolic exploitation of symmetry in numerical pathfollowing. Impact Comput. Sci. Engrg., 1991, 3, 330-365.

(35) Kuznetsov, Yuri. A., Elements of Applied Bifurcation Theory. Springer, 1995.

(36) Champneys, A. R. and Kuznetsov Yu. A., Numerical detection and continuation of codimension-two homoclinic bifurcations. Internat. J. Bifur. Chaos, 1994, 4, 795-822.

(37) Champneys, A. R., Kuznetsov, Yu. A. and Sandstede, B., HomCont: An AUTO86 driver for homoclinic bifurcation analysis, version 2.0. Technical report, CWI, Amsterdam, 1995.

(38) Henderson, M., Computing implicitly defined surfaces: two parameter continuation. IBM Research Report RC 18777, IBM Thomas Watson Research Center, Yorktown Heights, New York, 1993.

(39) Wicklin, F. J., Pisces: A platform for implicit surfaces and curves and the exploration of singularities. Technical Report GCG89, Geometry Center, University of Minnesota, Minneapolis, 1995.

(40) Kevrekidis, I. G. and Jolly, M. S., On the use of interactive graphics in the numerical study of chemical dynamics. In AIChE Annual Meeting, New York, paper 22c, 1987.

(41) Nusse, H. E. and Yorke, J. A., Dynamics: Numerical Explorations. Springer, 1994.

(42) Fenichel, N., Persistence and smoothness of invariant manifolds for flows. Indiana Univ. Math. J., 1971, 21(3), 193-226.

(43) Lorenz, J., Lyapunov-type numbers and torus breakdown: Numerical aspects and a case study. Technical report, Mathematics Department, University of New Mexico, 1996.

(44) Broer, H. W., Osinga, H. M. and Vegter, G., On the computation of normally hyperbolic 
invariant manifolds. In Progress in Nonlinear Differential Equations and Their Applications, Vol. 19, ed. H. W. Broer, I. Hovei jn and F. Takens. Birkhäuser, 1996, pp. 423447.

(45) Ermentrout, B., XPPAUT1.61-the differential equations tool. Technical report, Department of Mathematics, University of Pittsburgh, 1995.

(46) Stech, H., A numerical analysis of the structure of periodic orbits in autonomous functional differential equations. In Dynamics of Infinite Dimensional Systems, ed. S.-N. Chow, and J. K. Hale. Springer, Berlin, 1987, pp. 325-337.

(47) Doedel, E. J. and Leung P. C., Numerical techniques for bifurcation problems in delay equations. Cong. Num., 1982, 34, 225-237 (Proc. 11th Manitoba Conf. on Num. Math. and Comp., Univ. of Manitoba, Winnipeg, Canada).

(48) Cometto, L., Dahmen, M. and Giannakopoulos, F., On the number of $p$-zeroes of a quasi-polynomial with application to the stability and Hopf-bifurcation of systems of differential equations with delays. Technical report, Department of Mathematics, University of Cologne, 1996.

(49) Luzyanina, T., Engelborghs, K., Lust, K. and Roose, D., Computation, continuation and bifurcation analysis of periodic solutions of delay differential equations. Internat. J. Bifur. Chaos, to appear.

(50) Ascher, U. M. and Spiteri R. J., Collocation software for boundary value differentialalgebraic equations. SIAM J. Sci. Comput., 1995, 15, 938-952.

(51) Bank, R. E., PLTMG, A Software Package for Solving Elliptic Partial Differential Equations. SIAM, 1990.

(52) Shroff, G. M. and Keller H. B., Stabilization of unstable procedures: The recursive projection method. SIAM J. Numer. Anal., 1993, 30(4), 1099-1120. 